CIUDAD Y TERRITORIO

ESTUDIOS TERRITORIALES

ISSN(P): 1133-4762; ISSN(E): 2659-3254

Vol. LII, № 206, invierno 2020

Págs. 901-922

https://doi.org/10.37230/CyTET.2020.206.12

CC BY-NC 4.0

\title{
Análisis urbano del próximo sistema multi-aeropuerto de la ciudad de Bogotá (Colombia)
}

Óscar Díaz-OLARIaga Profesor-Investigador, Facultad de Ingeniería Civil, Universidad Santo Tomás (Bogotá, Colombia).

RESUMEN: El Aeropuerto Internacional El Dorado en la ciudad de Bogotá (capital de Colombia) comenzó operaciones al final de la década de 1950 y a los pocos años empezó un importante desarrollo urbano en su entorno que se mantiene hasta el presente. Por otro lado, y desde la liberalización del transporte aéreo en el país (1991), el tráfico aéreo en el aeropuerto ha sido muy relevante, lo que llevó a la ejecución de dos ampliaciones del aeropuerto. La proyección de la demanda exige, a corto plazo, nuevas y relevantes ampliaciones del aeropuerto, pero fuertes restricciones (falta de suelo disponible y razones de carácter ambiental) hacen imposible cualquier nueva expansión. Por ello, y desde hace un lustro, está en marcha el desarrollo de un nuevo aeropuerto, por ahora conocido como El Dorado II, a $15 \mathrm{~km}$ del actual aeropuerto (y que se estima inicie operaciones en 2027/2028), y que servirá a la ciudad de Bogotá y zona de influencia. Entonces, en el presente artículo se realiza el análisis, con un enfoque urbano/territorial, del próximo sistema multi-aeropuerto para la ciudad de Bogotá.

PALABRAS CLAVE: Sistema multi-aeropuerto; Desarrollo urbano/territorial; Transporte aéreo; Bogotá.

\section{Urban analysis of the next multi-airport system of the city of Bogotá (Colombia)}

ABSTRACT: El Dorado International Airport" in the city of Bogotá (capital of Colombia) started operations at the end of the 1950 s and also a few years later began an important urban development in its environment that continues to the present. On the other hand, and since the liberalization of air transport in the country (1991), air traffic has increased highly, which led to the execution of two extensions of the airport. The demand projection requires, in the short term, new and relevant extensions, but strong

Recibido: 16.12..2019; Revisado: 17.02.2020

Correo electrónico: oscardiazolariaga@usantotomas.edu.co; NN ORCID: https://orcid.org/0000-0002-4858-3677

El autor agradece los comentarios y sugerencias realizados por los evaluadores anónimos, que han contribuido a mejorar y enriquecer el manuscrito original 
restrictions (like the lack of available land and environmental reasons) make any new expansion impossible. For this reason, and since five years ago, the development of a new airport - now known as "El Dorado II" ( $15 \mathrm{~km}$ from the current airport, and which is estimated to start operations in 2027/2028)is in progress, which will serve the city of Bogotá and its area of influence. This article presents an analysis with an urban/territorial focus of the next multi-airport system for the city of Bogotá.

KEYWORDS: Multi-airport system; Urban/territorial Development; Air transport, Bogotá.

\section{Introducción}

uchos aeropuertos importantes a nivel mundial están congestionados, dicha congestión crea retrasos y una diversidad de problemas derivados tanto para la aeronave (o aerolínea) como para los pasajeros, y esto generalmente se considera un problema del transporte aéreo (ZHANG \& ZhANG, 2006; PeLs \& VERHOEF, 2004; BRUECKNER, 2002). Además, estos grandes aeropuertos, generalmente antiguos, construidos y operativos desde hace varias décadas (muchos incluso más de medio siglo), ahora están rodeados por comunidades (desarrollo urbano intensivo) que se ven afectadas negativamente por el ruido y la polución ambiental generada por la actividad aeroportuaria (KNIPPENBERGER \& WALL, 2010; Postorino, 2010).

A corto plazo algunos de los efectos de la congestión del tráfico y los problemas ambientales se pueden mitigar mediante una planificación y gestión activa. Los peores aspectos de la congestión del tráfico aéreo pueden reducirse mediante una batería de estrategias y regulaciones. Por ejemplo, aplicar tasas aeronáuticas más elevadas en los períodos de mayor demanda reorienta parte del tráfico a diferentes horarios o incluso a otros aeropuertos, lo que reduce los niveles máximos de congestión (Mun \& TERAJI, 2012). Alternativamente, el racionamiento del número de operaciones durante los períodos pico por mandato regulatorio puede tener un efecto similar. El problema de la contaminación acústica también puede ser mitigado por la utilización de patrones (o protocolos) en las maniobras de aterrizaje y despegue (ICAO, 2008). Y finalmente, la mitigación puede llegar hasta a la reducción total de las operaciones en horario nocturno.

Pero cuando la demanda sigue aumentando, y los pronósticos estiman que lo seguirá haciendo en el futuro, el aumento de la capacidad del aeropuerto se transforma en la solución inevitable a los problemas de la congestión del aeropuerto. La forma en que se debe construir una capacidad aeroportuaria adicional es un tema de política pública importante para los gobiernos locales, regionales e incluso nacionales. Entonces, la cuestión más relevante con respecto a la capacidad adicional de los aeropuertos principales es si la capacidad adicional del aeropuerto debe o puede desarrollarse en el aeropuerto principal o en otro aeropuerto (secundario), ya existente o por construir (si no existe), en la misma región o área metropolitana. Cuando esto último sucede se está en presencia de lo que se conoce como sistema multi-aeropuerto (DE NeUfVILLE, 1984).

Entonces, en el presente artículo se realiza un análisis, con enfoque urbano y territorial, del próximo sistema multi-aeropuerto para la ciudad de Bogotá (capital de Colombia) que estará constituido por el (actual) Aeropuerto Internacional de Bogotá-El Dorado y un futuro aeropuerto por ahora conocido como Aeropuerto El Dorado II. El Aeropuerto Internacional de Bogotá-El Dorado (en adelante BOG) comenzó operaciones al final de la década de 1950. A pesar de que el desarrollo de su tráfico, y por lo tanto de sus infraestructuras e instalaciones, fue inicialmente muy lento, a los pocos años dio comienzo un desarrollo urbano en su entorno, preponderantemente informal (es decir, no ordenado ni controlado), que prácticamente no ha cesado hasta el presente, y cercando, literalmente, al aeropuerto en tres de sus cuatro caras. A principios de la década de 1990 el transporte aéreo se liberaliza en el país lo que da pie, entre otros hechos, a la privatización de los aeropuertos del país; con ello la demanda de transporte aéreo empieza a crecer y se dispara a partir de mediados de la década del 2000 cuando BOG se privatiza (Díaz OlARIAGA, 2016, 2017). Desde esa época, 2006, por un lado, el aeropuerto ha experimentado dos grandes ampliaciones, y por otro, el entorno del aeropuerto ha visto un relevante $e$ incesante desarrollo urbano en su entorno. Ahora bien, debido a que las proyecciones de demanda de transporte aéreo indican que la misma seguirá en aumento (AEROCIVIL, 2014a), y como el aeropuerto ya no se puede ampliar / expandir más (como se explicará en detalle en secciones posteriores), la autoridad pública aeronáutica de Colombia ha puesto en marcha, hace ya casi un lustro, el proyecto de un nuevo aeropuerto para la ciudad de Bogotá, el cual estará fuera de la ciudad (a $15 \mathrm{~km}$ de actual aeropuerto). Con ello, y en aproximadamente siete $u$ ocho años, la ciudad contará con un sistema multi-aeropuerto. En definitiva, el análisis pondrá en relieve la problemática de desarrollo urbano (no controlado) en el 
entorno del actual aeropuerto, el impacto ambiental derivado, la obligatoriedad de proveer capacidad vía un nuevo aeropuerto y las necesarias consideraciones en la planificación del nuevo aeropuerto para que no se repitan los errores y problemas que sucedieron (y suceden) en el desarrollo de BOG.

Debido a que el presente artículo es de revisión la metodología del mismo se desarrolla de la siguiente manera. El marco conceptual está divido en cuatro grandes bloques: el primero ayuda a contextualizar la función y el papel de los aeropuertosy el transporte aéreo con la conectividad territorial (y su impacto en el propio desarrollo de los territorios) y en la globalización de las ciudades (ya que el caso de estudio escogido es una gran urbe de casi 8 millones de habitantes y ciudad capital de país); el contenido del segundo bloque presenta los elementos básicos de lo que se conoce como sistema multi-aeropuerto, de tal forma de ayudar al lector a la compresión del concepto; en la tercera parte del marco conceptual se presentan y exponen los fundamentos que dan lugar al desarrollo de este tipo de infraestructuras de transporte aéreo; y finalmente el marco conceptual se cierra exponiendo las ventajas y desventajas, en diferentes vertientes (técnica, de transporte aéreo, operativa, socio-económica, ambiental, urbana, etc.), de estos desarrollos aeroportuarios. Paso seguido se desarrolla en detalle el caso de estudio, esta sección se divide en dos grandes partes, la primera sobre el actual aeropuerto y la segunda sobre el proyectado nuevo aeropuerto. Sobre el actual aeropuerto se presenta su historia, evolución (operacional), desarrollo en contexto con la ciudad a la que sirve y su impacto (social, ambiental, urbano, etc.) sobre ésta, y se analiza la viabilidad de su (necesaria) expansión. Y en segundo lugar, se analiza y evalúa el proyectado nuevo aeropuerto, también en varias vertientes o dimensiones (desarrollo de la infraestructura, operaciones, uso del suelo, impacto ambiental previsto, conectividad terrestre, problemáticas existentes, etc.). Finalmente, el artículo se cierra con las oportunas y correspondientes conclusiones finales.

\section{Marco conceptual}

\subsection{El papel de los aeropuertos en la conectividad territorial y en la globalización de las ciudades}

En términos generales, puede entenderse la conectividad como una cualidad que surge y se desarrolla de la existencia de vínculos entre objetos y funciones que se interrelacionan. De esta manera, la representación física del concepto abstracto de conectividad es el de una estructura que está conformada por una red de corredores (terrestres, aéreos, marítimos/fluviales) que sirven para movilizar bienes y personas entre distintos puntos del territorio. Las características de esta red dependerán, en primer lugar, de los aspectos físicos o estructurales del territorio donde ésta se localiza, es decir, de las dificultades o facilidades que este territorio ofrece al despliegue de dicha red. Asimismo, las características de los flujos, en cuanto a movilidad, volúmenes y tipo de recursos movilizados son también elementos determinantes de la configuración de la red (FIGUEROA \& ROZAS, 2005). Y finalmente, la conectividad sirve a múltiples propósitos en los distintos ámbitos de la actividad económica y social de un país, los más típicos: a) conectividad para facilitar la actividad económica y productiva; $b$ ) conectividad para el desarrollo y la integración social; c) conectividad geopolítica (ejercicio de la soberanía); y d) conectividad estratégica (conexión de las regiones / territorios de un país con otros países) (ROzAs \& FIGUEROA, 2006).

Y entrando en el terreno de la conectividad aérea, muchos gobiernos formulan políticas públicas de transporte aéreo para, entre otros, mejorar la conectividad entre sus territorios; los argumentos para que el sector gubernamental lo haga son diversos, los más importantes: a) económico (desarrollo productivo, generación de empleo y riqueza, etc.), y b) socio-político (integración y cohesión territorial) (BURGHOUWT, 2017; BURGHOUWT \& ReDONDI, 2013). En lo que se refiere al primer argumento, el económico, se estima que el transporte aéreo es un factor habilitante para un desarrollo económico más amplio en una región (VAN DE VIJVER \& al, 2016). Un análisis de la bibliografía existente en la temática confirma que el transporte aéreo de pasajeros y el empleo (en diferentes sectores industriales) en las regiones urbanas están positivamente vinculados (GOETZ, 1992; LIU \& al., 2006; AlkaAbi \& Debbage, 2007; Button \& TAYlor, 2000; NeAl, 2012; Green, 2007; BrueCKNER, 2003). La razón de estas afirmaciones es la observación de que mejores servicios de transporte aéreo implican una mejor accesibilidad que alienta a las empresas a ubicarse en una región y estimula la expansión de los negocios existentes (CoOper \& Smith, 2005; ZAK \& GetZneR, 2016).

En otro orden, con el inicio de la desregulación y la liberalización de la industria de la aviación (finales de década de 1970), que generó un crecimiento superlativo de la demanda (aún en curso), muchos aeropuertos superaron su función de solo estaciones de transporte aéreo y se convirtieron en grandes nodos intermodales urbanos. La globalización, concepto que también impacto en los grandes centros urbanos, 
acompañó el proceso de transformación de los aeropuertos. Hoy en día, y en varias ciudades / regiones del mundo, ya se está evolucionado a conceptos como "ciudad aeroportuaria", "región aeroportuaria", "aerotrópolis", "corredor aeroportuario" o "airea" (conjunto de zonas urbanas desarrolladas, no necesariamente conexas o lindantes entre sí, del área metropolitana que están predominantemente influenciadas por el aeropuerto, o que, a la inversa, influyen directamente en el aeropuerto (KNIPPENBERGER \& WALL, 2010), dando a entender que en la actualidad los aeropuertos están generando desarrollo urbano y económico también a través de una relación sinérgica y simbiótica entre el desarrollo comercial e industrial en el entorno más próximo del aeropuerto (BOQUET, 2018; ApPold \& KASARDA, 2013). En otras palabras, se está en presencia de "ciudades globales" que reciben beneficios sustanciales de sus (cada vez más grandes) aeropuertos principales (AsHFORD \& al., 2011). El éxito de los desarrollos de los aeropuertos, en conjunción con el desarrollo urbano de la ciudad que los acoge, dependió de factores tales como la disponibilidad del espacio (suelo) para el desarrollo del aeropuerto, la ubicación (y conexión) del aeropuerto dentro de las redes de comunicación terrestre, la estructura socioeconómica de la región, el entorno institucional del gobierno local / regional / nacional, y el marco de planificación (política pública) (BANAI, 2017).

Volviendo al concepto de "ciudad global", la misma, y según algunos autores, es aquella que se puede considerar como un nodo importante en el sistema económico global. El concepto tiene sus raíces en la geografía y los estudios urbanos y se basa en la idea de que la globalización puede entenderse como una ciudad desarrollada en lugares geográficos estratégicos de acuerdo con una jerarquía de importancia para el funcionamiento del sistema global en términos financiero, empresarial, de comercio internacional, etc. (AsHFORD \& al., 2011). Se han realizado muchos estudios sobre este tema para definir el concepto de ciudad global e identificar sus atributos. Uno de los primeros intentos de definir y clasificar ciudades globales se realizó en 1998 por el Globalization and World Cities (GaWC) Research Network de la Universidad de Loughborough en el Reino Unido (GAWC, 2019a), ellos agruparon varios indicadores en cuatro grandes grupos característicos: a) económico, b) político, c) cultural, d) infraestructuras. En el presente, el ranking mundial de ciudades globales, según el GaWC, se divide, primero en cinco grandes grupos (alfa, beta, gama, alta suficiencia, suficiencia); el primero de ellos (y más importante) es el "alfa", y éste a su vez se divide en cuatro niveles de relevancia: alfa++, alfa+, alfa, y alfa-.
Mencionar que la ciudad de Bogotá, caso de estudio de la presente investigación, figura categorizada en el grupo alfa, nivel alfa-, según el GaWC 2018 (GAWC, 2019b).

\subsection{Sistema multi-aeropuerto: definición y conceptos básicos}

Un sistema multi-aeropuerto se define como un conjunto de dos o más aeropuertos dentro de una misma región metropolitana (BONNEFOY \& al., 2010; DE NeufVILLE, 1984). Esta definición implica varios condicionantes: a) los aeropuertos sirven, principalmente, tráfico comercial; b) el concepto de "región metropolitana" puede hacer referencia a un único municipio (o ciudad) o a varios (colindantes entre ellos), incluso puede que las unidades administrativas locales (los municipios) pertenezcan a diferentes unidades administrativas regionales; c) no tiene relevancia alguna el tipo de gobernanza o propiedad de los aeropuertos; d) por lo general los aeropuertos del sistema tienen relevancia en el volumen de tráfico que gestionan, más de un millón de pasajeros/año (independientemente si el tráfico es nacional y/o internacional); e) en un sistema multi-aeropuerto hay un solo aeropuerto al cual se lo considera como el principal (por lo general el que gestiona mayor volumen de tráfico), los demás se consideran secundarios (que suelen gestionar desde el $5 \%$ o $10 \%$, en adelante, del tráfico del aeropuerto principal); y f) los aeropuertos del sistema pueden operar de forma totalmente independientes entre sí o ser dependientes, es decir, compartir tráfico de conexión y/o fuerte interrelación de las operaciones (NASA, 2011).

\subsection{Origen y desarrollo de los sistemas multi-aeropuerto}

El crecimiento de los sistemas multi-aeropuerto en regiones metropolitanas puede explicarse haciendo un análisis desde tres vertientes: técnica, económica y política pública (DE NeufVILle \& Odoni, 2013; Fuellhart \& AL, 2013; Bonnefoy \& al., 2010; de NeufVILLE, 1995):

\section{Razones técnicas:}

A. Demanda / Capacidad: cuando la demanda en un aeropuerto se acerca a su máxima capacidad instalada (principalmente su capacidad de pista), la autoridad aeroportuaria local / regional / nacional estará más inclinada a dar respuesta a un aumento (previsto) de la demanda (y dar continuidad al 
crecimiento de las operaciones) en aeropuertos alternativos. Con esta solución la autoridad pública aeronáutica tratará de evitar un incremento desmedido de los retrasos en el aeropuerto principal; y para el caso de aeropuertos coordinados, evitará problemas en la gestión de las franjas horarias (slots).

B. Limitaciones/Restricciones: Ios aeropuertos principales pueden tener:

a. límites en el tipo o volumen de operaciones que puede admitir, por ejemplo debido a la configuración (operacional) de su sistema de pistas y/o campo de vuelo;

b. restricciones ambientales (debido principalmente a índices inadmisibles de contaminación acústica y/o de emisiones);

c. no disponibilidad (o congestión) del espacio aéreo relacionado;

d. limitaciones urbanas (no disponibilidad de suelo para ampliaciones del aeropuerto).

\section{Razones económicas:}

Ingreso de nuevos operadores aéreos, particularmente con interés en desarrollar nuevos mercados. Las aerolíneas de bajo costo, principales nuevos entrantes, requieren, en particular, bajos costos operativos para permitir el desarrollo (rentable) de sus modelos de negocio. Por lo tanto, pueden crecer operando en aeropuertos con bajos costos operativos a expensas de otras características, como la proximidad al mercado.

Sistema de conectividad y accesibilidad terrestre (vial, ferroviario) del nuevo sitio del aeropuerto a las áreas de captación geográficas (a quien servirá el aeropuerto).

III. Razones de política pública:

a. Incentivos (económicos) por parte de autoridades locales / regionales / nacionales con el objetivo de aumentar su conectividad regional / global de los aeropuertos de la ciudad / región.

b. Motivaciones y/o necesidades de otras industrias que demandan nuevas infraestructuras aéreas para su desarrollo, principalmente el turismo y el comercio exterior.

c. Factores externos (geo-políticos, coyuntura económica regional / global, etc.).

Los tres factores descritos suelen estar, a menudo, altamente interrelacionados. En particular, la relación entre la demanda de operaciones (despegues y aterrizajes) y la capacidad del aeropuerto afecta a varios de los factores descritos anteriormente y es un poderoso motor del crecimiento de las operaciones de las aerolíneas en los aeropuertos secundarios. A medida que la demanda se acerca a la máxima capacidad instalada del aeropuerto aumentan los retrasos en los vuelos, y los costos de operación de la aerolínea aumentan con los retrasos. Al mismo tiempo, la satisfacción de los pasajeros se reduce a medida que aumenta el tiempo de viaje, lo que reduce la demanda de pasajeros de las aerolíneas y, por lo tanto, los ingresos (BEZERRA \& Gomes, 2019; VASIGH \& al., 2013). Con el fin de mitigar los efectos negativos de las limitaciones de capacidad del aeropuerto, es decir, el aumento de los costos operativos y la disminución de la demanda de pasajeros, los operadores aeroportuarios pueden considerar el crecimiento de las operaciones en aeropuertos alternativos que presenten menos (o nula) congestión. Este resultado es mejorado por los operadores de estos aeropuertos alternativos, que pueden reducir las tarifas de aterrizaje e introducir otros incentivos, reduciendo aún más el costo de operar allí. Una estrategia de este tipo puede ser particularmente atractiva para los operadores aéreos de bajo costo, que requieren bajos costos operativos para ejecutar sus modelos de negocios (BUDd \& Ison, 2017).

Para algunas ciudades / regiones (o incluso países) se ha demostrado que el desarrollo de sistemas multi-aeropuerto permite que el crecimiento del sistema de transporte aéreo se amplíe a nivel regional o nacional, incluso cuando los aeropuertos individuales en una ciudad / región alcanzan su máxima capacidad. Por ello, un aeropuerto secundario ya existente (con posibilidades de ampliación / expansión), o un nuevo aeropuerto (por desarrollar), son un requisito necesario para que esto ocurra (Bonnefoy \& HANSMAN, 2007).

Entonces, cuando la demanda de pasajeros aéreos en un aeropuerto alcanza su máxima capacidad, o así lo anticipan los pronósticos (en el corto o medio plazo), existen tres respuestas posibles para absorber dicha futura demanda: a) aumentar la capacidad del aeropuerto, construyendo infraestructura como pistas, calles de rodaduras, plataformas, edificios terminales, otras instalaciones, etc.; b) modificando la configuración (y estrategia) de las operaciones aéreas (despegues y aterrizajes); o c) trasladar o derivar la actividad (parcial o total) a otro aeropuerto geográficamente próximo o cercano, si el mismo no existe implicará entonces la construcción de uno nuevo (Postorino \& PRATICO, 2012).

$Y$ finalmente, el desarrollo de sistemas multiaeropuerto plantea varios desafíos en términos 
de planificación y desarrollo. La evolución de estos sistemas generalmente ocurre en términos de medio o largo horizonte temporal (lustro o incluso una década) e involucra a múltiples partes interesadas (pasajeros, aerolíneas, operadores de aeropuertos, autoridades aeronáuticas, entes reguladores, autoridades administrativas locales / regionales / nacionales, etc.) (ZIETSMAN \& VANDERSCHUREN, 2014).

\subsection{Ventajas y desventajas de los sistemas multi-aeropuerto}

Es cierto que los sistemas multi-aeropuerto pueden presentar algunas desventajas, como por ejemplo (DE NeufVILLE \& Odoni, 2013; BonNeFoy \& al., 2010):

- la segmentación de las operaciones en varios aeropuertos limita las economías de escala para las aerolíneas;

- no hay suficiente tráfico en el (nuevo) aeropuerto secundario, o al menos no lo previsto en el plan maestro del aeropuerto, lo que no permite al operador del aeropuerto amortizar, en el plazo previsto, la gran inversión realizada;

- la posibilidad limitada de que los pasajeros se conecten entre vuelos en los diferentes aeropuertos que sirven a la región metropolitana;

- multiplicidad de inversiones en infraestructuras de accesibilidad (terrestre);

- multiplicidad de afectaciones ambientales;

- impacto múltiple en la desvalorización del suelo (en el próximo entorno de los aeropuertos);

- conflicto múltiple en la planificación urbana (o territorial) a medio, largo y muy largo plazo.

Pero por otro lado los sistemas multi-aeropuerto ofrecen ventajas significativas, a saber (dE NEUFVILLE \& ODONI, 2013; FASONE \& al., 2012; MARTIN \& Voltes-DortA, 2011; Bonnefoy \& al., 2010):

- aliviar la congestión en los aeropuertos principales al tiempo que proporciona capacidad adicional al sistema de transporte aéreo local / regional;

- proporcionar una mayor robustez operativa mediante el desacoplamiento espacial de las operaciones y la reducción de los efectos de las interrupciones;

- ofrecer nuevas alternativas de viaje para los residentes de la región metropolitana, lo que se traduce en una reducción en la distancia de acceso al aeropuerto y el tiempo de viaje;

- generar positivos impactos económicos regionales directos, indirectos e inducidos (es decir, empleo, fuentes de ingresos para las ciudades circundantes a partir de impuestos, atracción de nuevas empresas, etc.);

- mejorar la conectividad local / regional: a diferencia de los aeropuertos periféricos (muy alejados del centro urbano), los aeropuertos cercanos a las grandes regiones metropolitanas (o inmersos en las mismas) tienen mejores conexiones con los sistemas de transporte público de alta capacidad; dichas conexiones se complementan con las redes viales que facilitan el acceso al aeropuerto y permite a éste aumentar su capacidad de "captación" geográfica, incluso más allá de la propia región metropolitana; todo ello beneficia, desde el punto de vista de las comunicaciones / conectividad, a los habitantes de la región metropolitana:

- reducir los efectos de las posiciones monopolísticas que en algún momento pueden surgir, cuando en la ciudad o región existe un solo aeropuerto (en otras palabras, incentivar la competencia aeroportuaria).

\section{Caso de estudio a: el aeropuerto de Bogotá El Dorado}

\subsection{Características generales del aeropuerto}

El Aeropuerto Internacional de Bogotá-El Dorado (H24, código IATA: BOG; código OACl: SKBO), situado en la ciudad de Bogotá D.C. (ciudad capital de Colombia), y único aeropuerto que sirve a la ciudad, es de propiedad pública pero con operación concesionada desde el año 2007 al consorcio (privado) OPAIN, S.A. (Díaz Olariaga, 2017), año en que el aeropuerto experimentó el inicio de una importante ampliación de su infraestructura e instalaciones (principalmente el nuevo edifico terminal de pasajeros), con una inversión de USD 650 millones, y que culminó en el año 2013. En el año 2015 dio inicio una segunda ampliación que finalizó en el año 2018, esta última ampliación implicó actuaciones tanto en el "lado tierra" como en el "lado aire".

El aeropuerto (situado a $2.548 \mathrm{~m}$ sobre el nivel del mar) cuenta con un área aproximada de $975 \mathrm{ha}$, se encuentra a $12 \mathrm{~km}$ del centro de la ciudad de Bogotá, y limita con las localidades de 


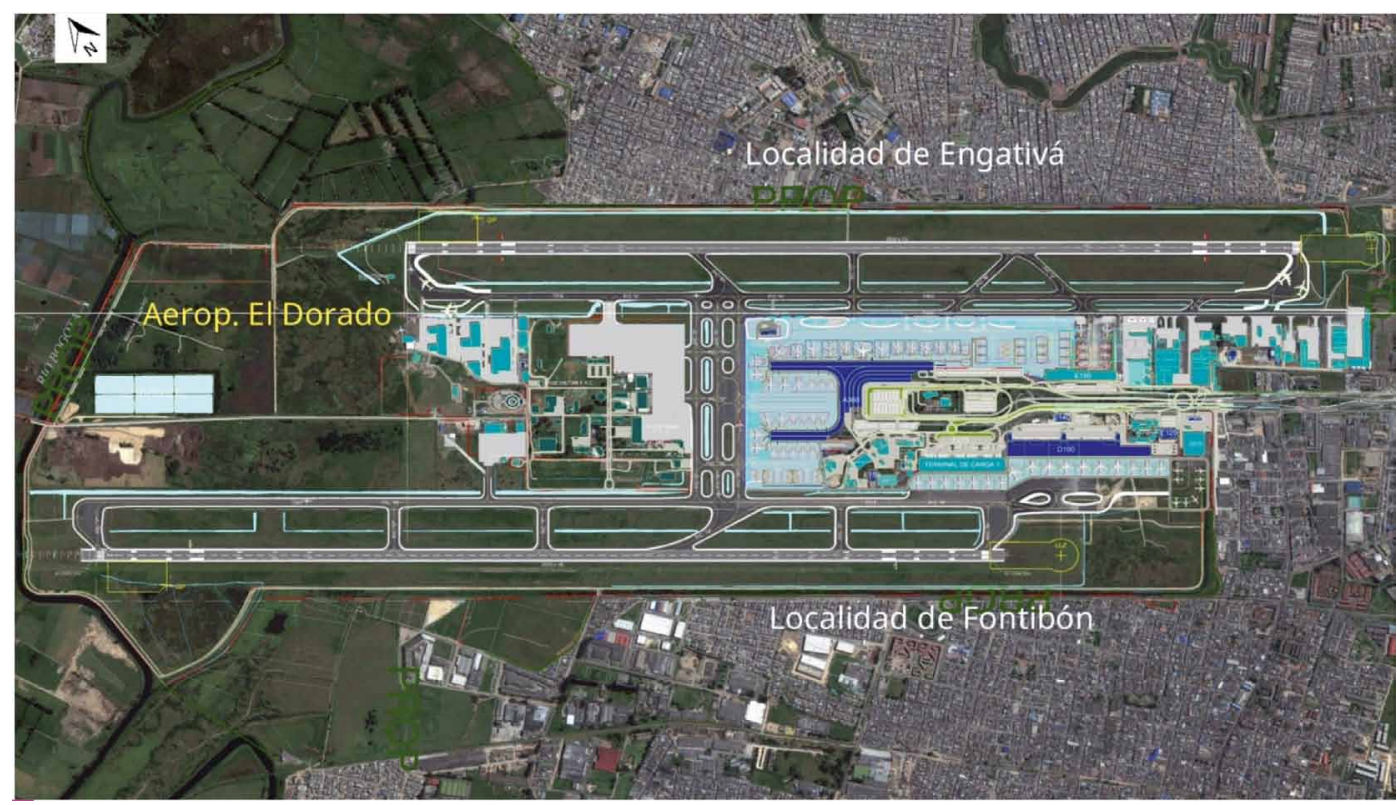

FIG. 1/ Aeropuerto Internacional de Bogotá-El Dorado.

Fontibón y Engativá (en sus caras sur y norte respectivamente y también en toda la cara oriental del aeropuerto), ambas pertenecientes, administrativamente, a la ciudad de Bogotá, y por el río Bogotá y humedales pertenecientes a la zona rural de la ciudad de Funza en su cara occidental (ver FIG. 1). El aeropuerto cuenta con un sistema de dos pistas paralelas, 13L/31R (pista norte) y $13 R / 31 L$ (pista sur), ambas para operaciones de despegue y aterrizaje, y cada una con una longitud y ancho de $3.800 \times 45$ m (AIP, 2019). Las estadísticas para el año 2018 indican que el aeropuerto movilizó 32,7 millones de pasajeros totales (nac. + int.), 741 mil toneladas de carga aérea totales y gestionó 333 mil operaciones (despegues / aterrizajes) totales (AEROCIVIL, 2020). En el aeropuerto trabajan, aproximadamente, unas 23.000 personas (DíAz OlaRIAgA \& al., 2018b).

\subsection{Desarrollo operacional del aeropuerto: evolución y perspectiva futura}

BOG ha liderado e impulsado el desarrollo del tráfico aéreo a nivel nacional en las últimas dos décadas y media; desde el año de la liberalización del transporte aéreo en el país (1991) al año 2018 el tráfico total de pasajeros creció más de siete veces $(711 \%)$ (AEROCIVIL, 2020). Este superlativo crecimiento del tráfico, y de la demanda, fue absorbido de forma paulatina en el tiempo, y muy especialmente por las dos últimas ampliaciones del aeropuerto, 2007-2013 y 2015-2018 (ver FIG. 2 a,b,c). Ahora bien, estas ampliaciones no implicaron actuación alguna en el sistema de (dos) pistas del aeropuerto.

A nivel regional, y desde hace al menos una década, BOG se ha transformado en un aeropuerto de referencia y que se consolida como tal gracias a la gran dinámica en el crecimiento de su tráfico. Como lo muestran los indicadores que se presentan a continuación, BOG no solo es un "hub" nacional sino que aspira a ser un "hub" regional. Entonces, en Latinoamérica BOG lleva varios años consolidado como el tercer aeropuerto por número de pasajeros movilizados y primero por volumen de carga aérea transportada (ACI-AIRPORT Council InTERnATIONAL, 2019; Díaz Olariaga \& ÁVILA, 2015). En lo que se refiere a la conectividad internacional, en el periodo 1992-2018 BOG ha duplicado los destinos internacionales directos (actualmente más de 60 conexiones internacionales directas), principalmente con ciudadesdestinos en las Américas y en Europa (AERociviL, 2020). Según un informe reciente (IATA, 2019) la ruta Bogotá (EI Dorado)-Madrid(Barajas) es la novena más importante, por volumen de pasajeros transportados, de entre todas las existentes entre Latinoamérica \& Caribe (L\&C) y Europa; y dentro de la región latinoamericana, las rutas Bogotá (El Dorado)-Ciudad de México (Benito Juárez) y Bogotá (El Dorado)-Lima (Jorge Chávez) son la séptimay décima, respectivamente, por volumen 


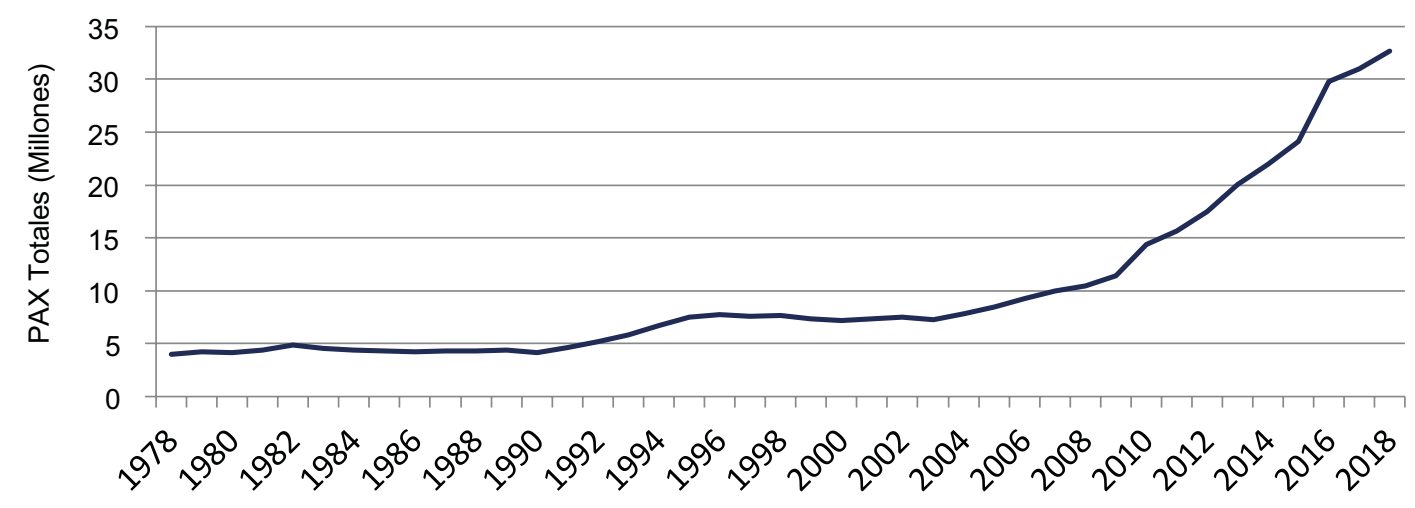

Figura A

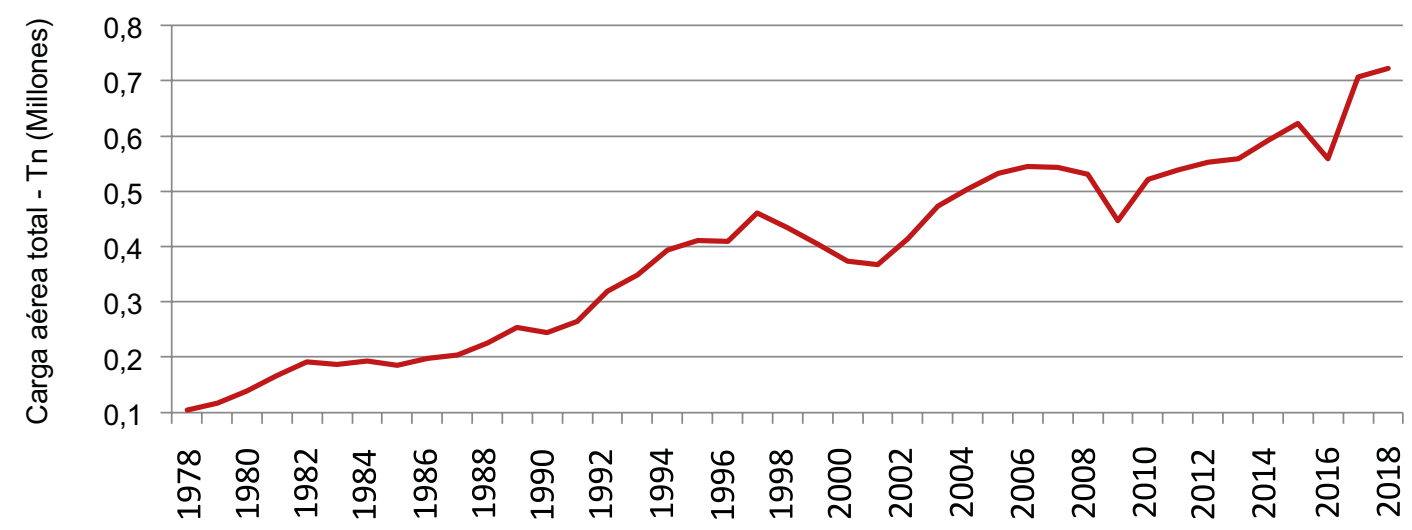

Figura B

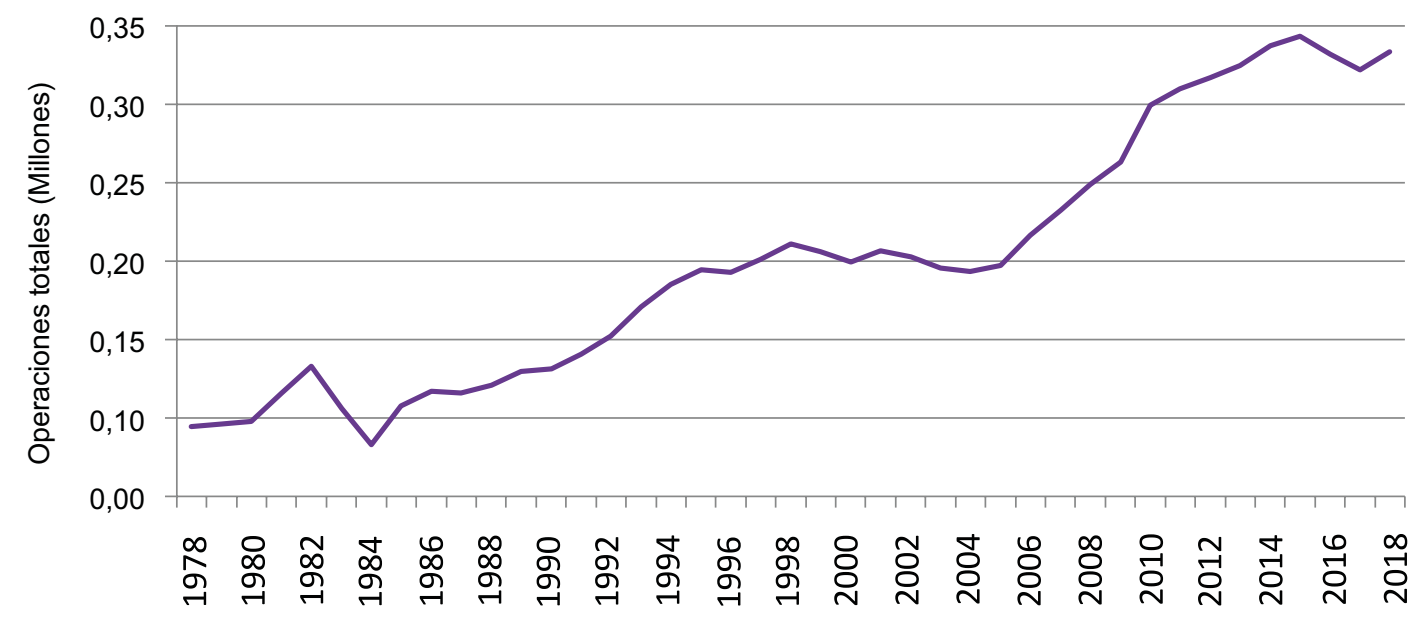

Figura C

FIG. 2 ( $A, B, C)$ / Desarrollo del tráfico en BOG, periodo 1978-2018.

Fuente: AEROCIVIL, 2020. 
de pasajeros transportados de entre todas las rutas del subcontinente. Este comportamiento, y tendencia, de BOG va en la misma línea y es coherente con el dinámico desarrollo del transporte aéreo en Latinoamérica, región, que en 2018, y a nivel mundial, ocupa el tercer lugar por crecimiento del RPK (Revenue Passenger Kilometers) (7\%) y el primer lugar en FTK (Freight Tonne Kilometres) (7,5\%); y donde las rutas entre L\&C y América del Norte, como dentro del mismo subcontinente ( $L \& C)$, se encuentran de entre las de mayor crecimiento a nivel global (IATA, 2019). Se podría afirmar que este desarrollo es una lógica consecuencia de las políticas públicas de inversión, donde solo en el periodo 2008-2015 los países latinoamericanos invirtieron USD 20 mil millones en infraestructura aeroportuaria, inversión anual promedio de $0,05 \%$ del PIB regional (CEPAL, 2019).

En otro orden, la capacidad de un aeródromo viene condicionada por la capacidad de su sistema de pistas, y ésta se mide como la cantidad de operaciones (despegues y aterrizajes) por hora que el sistema puede absorber o gestionar dentro de aquellos límites que garantizan la seguridad operacional (KAZDA \& CAVES, 2015; HORONJEFF \& al., 2010; JANIC, 2009). Entonces, la capacidad

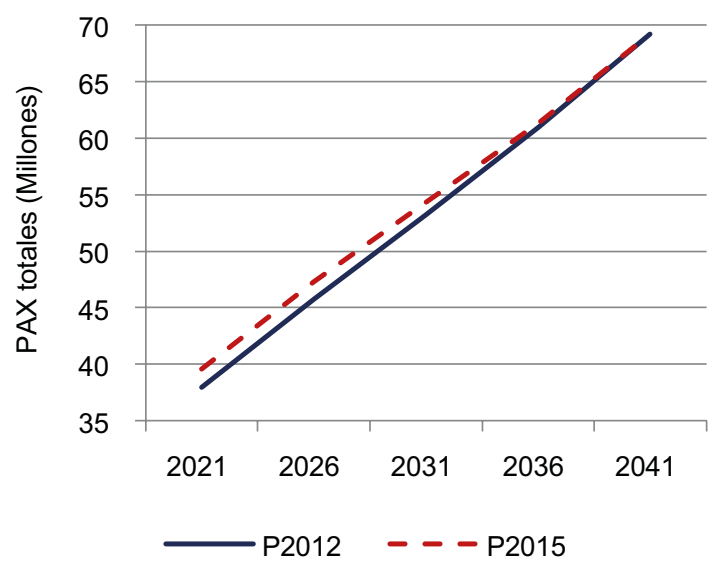

declarada del sistema de (dos) pistas de BOG es de 82 operaciones/hora (AIP, 2013). Por otro lado, la demanda no siempre es la misma a toda hora (y todos los días) sino que existen horas picos y horas valle (de mínima demanda). Actualmente la demanda en horas picos (con respecto a la capacidad declarada) es de $125 \%$ para operaciones de despegue y del $110 \%$ para operaciones de aterrizaje (AEROCIVIL, 2020). Proyecciones de demanda y capacidad requerida, realizadas en las dos últimas actualizaciones del Plan Maestro del aeropuerto (2012 y 2015), se muestran en la FIG. 3 y FIG. 4.

Como se aprecia en las proyecciones de la FIG. 4, a partir del año 2021 sería necesaria la construcción de una nueva pista para poder dar respuesta a la demanda proyectada, e incluso más pistas (hasta disponer de un sistema de 5 pistas) en el largo plazo (2041) si se pretende no frenar el crecimiento del aeropuerto; y este aumento del campo de vuelo (pistas, calles de rodadura, etc.) conduciría al obligado desarrollo de nuevas plataformas, edificios terminales asociados y otras instalaciones relacionadas necesarias. Pero como se explicará en un apartado posterior la factibilidad de expandir el aeropuerto (principalmente su sistema de pistas) es prácticamente inviable.

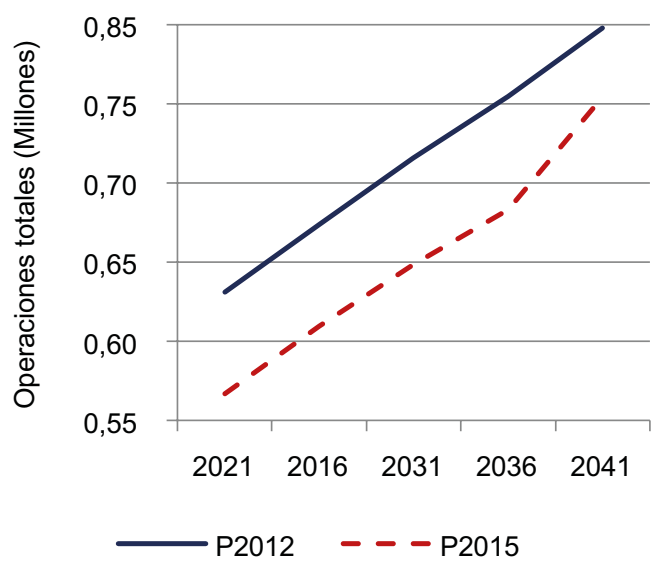

Fig. 3/ Proyección de la demanda según pronósticos de 2012 (P2012) y de 2015 (P2015).

Fuente: AerociviL, 2014a, 2016.

\begin{tabular}{lccc}
\hline Periodo & Demanda de operaciones (hora pico) & Capacidad requerida & Pistas necesarias \\
\hline $\mathbf{2 0 2 1 - 2 0 2 6}$ & 120 & $118-124$ & $2-3$ \\
\hline $\mathbf{2 0 2 6 - 2 0 3 1}$ & 137 & $136-154$ & $3-4$ \\
\hline $\mathbf{2 0 3 1 - 2 0 4 1}$ & 154 & $186-196$ & $4-5$
\end{tabular}

Previsiones de demanda de tráfico y capacidad requerida. 


\subsection{Evolución del desarrollo \\ urbano en el entorno \\ del aeropuerto}

El 10 de diciembre de 1959 se inauguró el Aeropuerto de Bogotá-El Dorado. Por entonces, el aeropuerto estaba totalmente aislado de la ciudad. En la década de 1960 se presentó un crecimiento urbano incipiente en el entorno del aeropuerto, tanto formal como informal (es decir sin obedecer a ningún tipo de planificación ordenada del territorio). De hecho, en algunas zonas aledañas se empezaban a conformar pequeños núcleos de actividades principalmente residenciales, que no seguían la línea de una ciudad planificada. Simplemente se entenderían como decisiones de localización particulares y dispersas sobre el territorio (SÁNCHEZ, 2011).

Entrada la década de 1970 el entorno aeroportuario continuaba consolidándose de manera acelerada, el desarrollo urbano importante empezó en el lateral sur del aeropuerto, el desarrollo urbano informal seguía siendo persistente. Entrada la década de 1980 el desarrollo urbano seguía intenso en el lateral sur del aeropuerto, pero también contagió su dinámica tanto en el lateral norte como oriental. Para mediados de la década de 1990 el desarrollo urbano, tanto formal como informal, había, literalmente, cercado totalmente el aeropuerto en sus laterales sur, norte y oriental (recordar que el lateral occidental del aeropuerto no tiene desarrollo urbano (ver FIG. 1) (CCB, 2008a). Para el año 2005 la población en las localidades que circundan el aeropuerto era de 1 millón de habitantes (DANE, 2019). Destacar que incluso en el presente sigue en curso una urbanización de las zonas antes mencionadas y con un importante porcentaje de informalidad. Este modelo de ocupación, con un relevante índice de informalidad, y nunca oportunamente controlado, ha restringido, en todo este tiempo, el margen de planificación y de acción tanto del aeropuerto como de la misma ciudad (ALCALDía DE BOGOTÁ, 2018).

La ejecución del proyecto de la segunda pista del aeropuerto (año 1995) requirió de una licencia ambiental. Esta normativa definió algunas restricciones de operación con el fin de salvaguardar los intereses de los residentes que se encontraban ubicados en el territorio aledaño. Sin embargo, estas medidas fueron paliativas, pues el problema nunca se resolvió de la manera adecuada. El proceso de mitigación del ruido fue ineficaz, y además se afectó sustancialmente el punto óptimo de utilización del aeropuerto, y por ende su productividad y competitividad. En otras palabras, se redujo la calidad de vida de los habitantes por cuenta del problema de salud ocasionado y se dejaron de percibir utilidades y beneficios en el sector aeronáutico y en los demás sectores de la economía que se ven beneficiados por la actividad aeroportuaria (Díaz OLARIAGA, 2018; CCB, 2008b).

En 1999 la administración local de la ciudad expide un decreto mediante el cual se define el área de influencia aeronáutica y aeroportuaria. En esta normativa se restringen los usos residenciales en las inmediaciones del aeropuerto con base a los impactos de la operación aeronáutica en materia de contaminación acústica y presión sonora. No obstante, a pesar de las restricciones en el modelo de ocupación, la conurbación producida en esta zona de la ciudad se entendía como un fenómeno tangible y concreto sobre el territorio, por lo que dicho marco normativo no contó con la aplicabilidad requerida. Esta situación ha dejado en entredicho la capacidad de planificar tanto de la administración local como de la autoridad pública aeronáutica (propietaria del aeropuerto) bajo un proceso concertado entre los diferentes actores involucrados que permitiera incrementar tanto los niveles de calidad de vida de los residentes vecinos del aeropuerto como el nivel de competitividad del mismo (Alcaldía de Bogotá, 2018).

En la década del 2000 las diferentes administraciones de la ciudad de Bogotá empezaron a formular estrategias urbanísticas con el objetivo de adelantar intervenciones sobre las áreas del entorno del aeropuerto para configurar espacios de gran accesibilidad y calidad ambiental que incentivaran el desarrollo de actividades empresariales, y de servicios aeroportuarios y logísticos, aprovechando las ventajas comparativas de esa localización. Pero ninguna de dichas estrategias logró materializarse. Múltiples conflictos de intereses impidieron desarrollar un instrumento que armonizara y articulara la operación aeroportuaria con el territorio aledaño. En el año 2007 el estado nacional, a través de un ente delegado y coordinando intensiones e intereses a nivel local, regional y nacional, expidió una norma (DNP, 2007) mediante la cual se establecía la estrategia institucional para el desarrollo de lo que se denominó Macroproyecto UrbanoRegional del Aeropuerto El Dorado de Bogotá (CEDINS, 2011), cuyo objetivo era promover el desarrollo armónico y planificado del área de influencia del aeropuerto a través de acciones y actuaciones público-privada concertadas. En otras palabras, con dicha iniciativa se pretendía sentar las bases para el desarrollo del entorno del aeropuerto bajo el concepto de ciudad aeroportuaria o aerotrópolis. Sin embargo, y debido a conflictos de intereses entre todos los actores implicados, este proyecto no se logró desarrollar ni materializar. 
Finalmente, en el año 2011, la administración local de la ciudad de Bogotá formula un nuevo documento técnico de soporte para la operación estratégica del entorno urbano del aeropuerto. Sin embargo, en esta ocasión la viabilidad financiera de la operación fue limitada, pues no se cuantificaron los costos de las alternativas de solución que se plantearon, y por tanto tampoco se definió un plan de intervenciones e inversiones a corto, mediano y largo plazo, con su respectiva fuente de financiación (ALCALDIA DE BOGOTÁ, 2018).

\subsection{Conectividad del aeropuerto con la ciudad}

En términos de movilidad, la Av. Calle 26, o también conocida como Av. El Dorado, es la única vía principal de acceso al aeropuerto. Esta importante arteria vial fue construida de forma simultánea con el aeropuerto (inicia servicio en el año 1958, un año antes que el aeropuerto), y tiene una longitud de $13,3 \mathrm{~km}$ y nace en el centro de la ciudad y termina en el mismo aeropuerto. Para el año 2015 el área de influencia del aeropuerto es el destino de por lo menos $1,17 \mathrm{mi}-$ llones de viajes en día hábil y 719 mil en día no hábil (SDM, 2015).

El aeropuerto tiene un solo modo de acceso, el vial, y durante cinco décadas los medios de acceso, en transporte público, fueron el bus convencional (de línea) y el taxi. Es recién en el año 2012 cuando entra en operación una línea troncal (a lo largo de la Av. Calle 26) del sistema BRT (Bus Rapid Transit) de la ciudad de Bogotá. $Y$ asimismo el sistema BRT no llega al terminal aéreo, sino que termina en una gran estación de intercambio a $3 \mathrm{~km}$ del aeropuerto. De allí, buses alimentadores del sistema llevan a los usuarios al aeropuerto y viceversa (usando los carriles viales de uso mixto de las vías que conducen al aeropuerto) (DIAZZ OlariagA \& al., 2018b).

Una investigación realizada a principios de 2017 (Díaz Olariaga \& al., 2018a; 2018b), sobre la accesibilidad al aeropuerto en estudio, determinó, entre otras cosas, que el $72,6 \%$ de las personas que acceden al aeropuerto, ya sean trabajadores / empleados del aeropuerto (e instalaciones anexas) o viajeros (pasajeros aéreos), utiliza el transporte público masivo (es decir, el sistema BRT).

El relevante crecimiento del tráfico aéreo que gestiona el aeropuerto, sumando a ello el dinámico desarrollo urbano en el entorno del mismo (como ya se ha explicado en una sección anterior) conduce a concluir que la situación actual de conectividad ciudad-aeropuerto, a través de una única vía principal (y de un solo modo de transporte, el vial), se presenta como un serio limitante, tanto para el mismo crecimiento del aeropuerto (generando un verdadero "cuello de botella") como para los potenciales productivos de la zona de influencia del aeropuerto.

\subsection{Desarrollo ambiental del entorno del aeropuerto}

Las estrategias y acciones en materia de mitigación del ruido en el aeropuerto en estudio dieron comienzo recién en el año 1998 cuando la autoridad pública aeronáutica de Colombia desarrolló el "Manual de Procedimientos para Abatimiento de Ruido del Aeropuerto Internacional de Bogotá-El Dorado" (posteriormente actualizado en el año 2008) (AEROCIVIL, 2008). Por lo que hasta el año 1998, y muy en especial en la década de 1990, donde el crecimiento del tráfico empezó a ser relevante, la contaminación acústica en el entorno próximo del aeropuerto no tenía ningún tipo de plan para su mitigación ni control, por lo que la afectación (en la salud y bienestar) en los vecinos del entorno empezaba a ser importante (Díaz Olariaga, 2018).

La puesta en práctica de las estrategias de mitigación de la polución acústica dio sus buenos resultados, ya que en el periodo 2002-2014, a medida que aumentaban las operaciones aéreas en el aeropuerto ( $120 \%$ en el periodo) el número de personas expuestas disminuía (en un $40 \%$ en el mismo periodo), mientras que la población de las localidades que rodean el aeropuerto aumentaba en aproximadamente un $180 \%$ (AEROCIVIL, 2014b, 2009, DANE, 2019). Pero, así y todo, en el año 2014 la población expuesta, en el área de influencia directa del aeropuerto, era de 147.455 personas (en un área aproximada de $7,75 \mathrm{~km}^{2}$ ), los datos de exposición se muestran en la FIG. 5 y en la FIG. 6.

\begin{tabular}{cr}
\hline Indicador & $\begin{array}{c}\text { N. personas expuestas } \\
\text { (\% del total) }\end{array}$ \\
\hline $65-70 \mathrm{~dB}(\mathrm{~A})$ & $105.731(71,7 \%)$ \\
\hline $70-75 \mathrm{~dB}(\mathrm{~A})$ & $34.715(23,54 \%)$ \\
\hline $75-80 \mathrm{~dB}(\mathrm{~A})$ & $7.004(4,75 \%)$ \\
\hline $80-85 \mathrm{~dB}(\mathrm{~A})$ & $5(0,003 \%)$ \\
\hline
\end{tabular}

FIG. 5/ Personas expuestas, según indicador Ldn, en el área de influencia directa del aeropuerto con uso residencial del suelo (año 2014).

Fuente: AEROCIVIL, 2014b. 


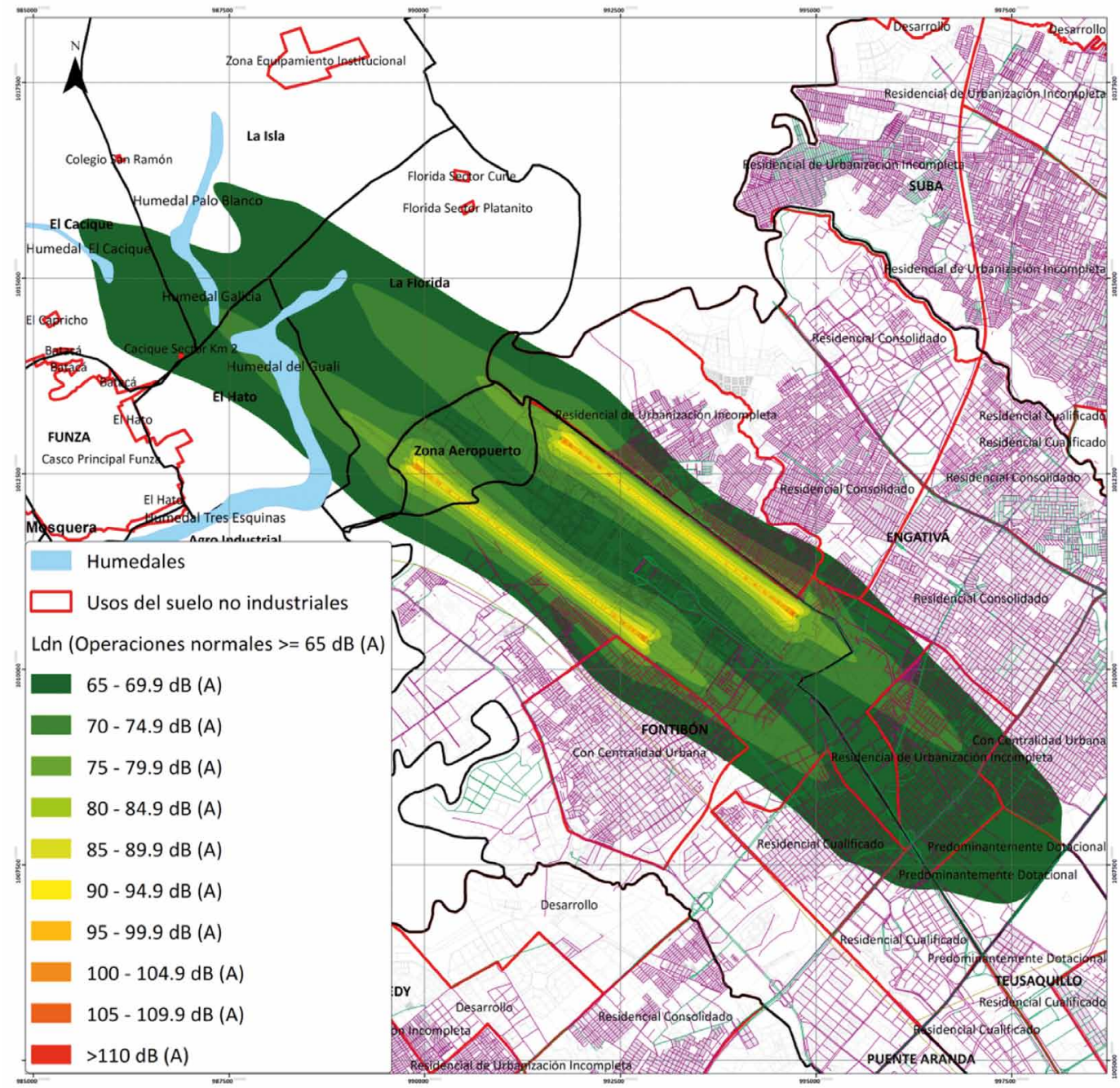

FIG. 6/ Áreas expuestas a valores de nivel de presión sonora iguales o mayores a $65 \mathrm{~dB}(\mathrm{~A})$ (año 2014).

Fuente: AEROCIVIL, 2014b.

\subsection{Inviabilidad de ampliación del aeropuerto}

Como se mencionó con anterioridad la capacidad de un aeródromo viene determinado por la capacidad de su sistema de pistas (KAZDA \& Caves, 2015; Horonjeff \& al., 2010; Janic, 2009). En el caso del Aeropuerto El Dorado el mismo ya está próximo a alcanzar su máxima capacidad operativa, ya que las ampliaciones realizadas (2007-2013 y 2015-2018) se enfocaron, casi exclusivamente, en su (nuevo) edificio terminal de pasajeros y accesos (lado tierra), con obras menores en su lado aire (campo de vuelo), en otras palabras, las ampliaciones no incluyeron nuevas pistas. Pero como lo indican las proyecciones de la demanda (ver FIG. 3 y FIG. 4), y para poder dar respuesta a la misma, se torna imperativo la construcción de la tercera pista en el corto plazo, la cuarta pista en el medio plazo y la quinta pista en el largo plazo. Ahora bien, como se puede apreciar en la FIG 7, es prácticamente imposible la construcción de nuevas pistas, ya que no hay disponibilidad de suelo, ni dentro del recinto aeroportuario, ni fuera (donde el desarrollo urbano ha cercado, literalmente, el aeropuerto). En la FIG. 7 se identifican zonas o áreas de oportunidad (en color verde) para ampliaciones del aeropuerto, pero las mismas sólo servirían para el desarrollo de instalaciones y/o infraestructuras de soporte, pero no para otra(s) pista(s) de por lo menos la misma longitud de las existentes $(3.800 \mathrm{~m}$., exigido debido a la altitud a la que se encuentra 
el aeropuerto). Y para mayor dificultad, en lo que refiere a disponibilidad de espacio, la zona identificada como "instalaciones militares" es una gran área del recinto aeroportuario ocupada por plataformas de todas las fuerzas armadas colombianas, que están allí casi desde la construcción del aeropuerto y que, en principio, allí seguirán, por lo que habría que descartar esa zona como de "oportunidad".

Entonces, ante la inviabilidad de ampliar el sistema de pistas del actual aeropuerto, en el año 2016 la autoridad pública aeronáutica de Colombia aprueba el "Plan Maestro del Aeropuerto El Dorado II", con el cual se da inicio al proyecto de desarrollo de un nuevo aeropuerto que servirá a la ciudad de Bogotá y región anexa (AEROVICIL, 2016). Los estudios situaron al nuevo aeropuerto fuera de la ciudad de Bogotá, aproximadamente a $15 \mathrm{~km}$ (hacia el oeste) del actual aeropuerto, entre los municipios de Madrid y Facatativá en la región administrativa de Cundinamarca (la ciudad de Bogotá se encuentra en el corazón de esta región, pero no forma parte del mismo desde el punto de vista político-administrativo, ya que tiene estatus de Distrito Especial). En siguientes apartados se presentará y analizará en detalle este nuevo aeropuerto.

\section{Caso de estudio B: el futuro aeropuerto el Dorado II}

\subsection{Características generales del nuevo aeropuerto}

Como ya se mencionó en una sección anterior, teniendo en cuenta las proyecciones de crecimiento de BOG, las limitaciones a la expansión / ampliación de las actuales instalaciones e infraestructuras, y las restricciones a las operaciones (despegues/aterrizajes) para mitigar los problemas de ruido que afectan las áreas residenciales vecinas, la autoridad pública aeronáutica puso en marcha, en el año 2015, todos los estudios necesarios para el proyecto y construcción de un nuevo aeropuerto, que debe servir a la ciudad de Bogotá y zona de influencia. En el año 2016 se contrataron los análisis de pre-factibilidad para determinar la viabilidad técnica, operacional y la elaboración del Plan Maestro del Nuevo Aeropuerto El Dorado II (AERocIVIL, 2016).

Para la ubicación y desarrollo del nuevo aeropuerto había un conjunto de supuestos, de entre los cuales se pueden destacar los siguientes (AEROCIVIL, 2016):

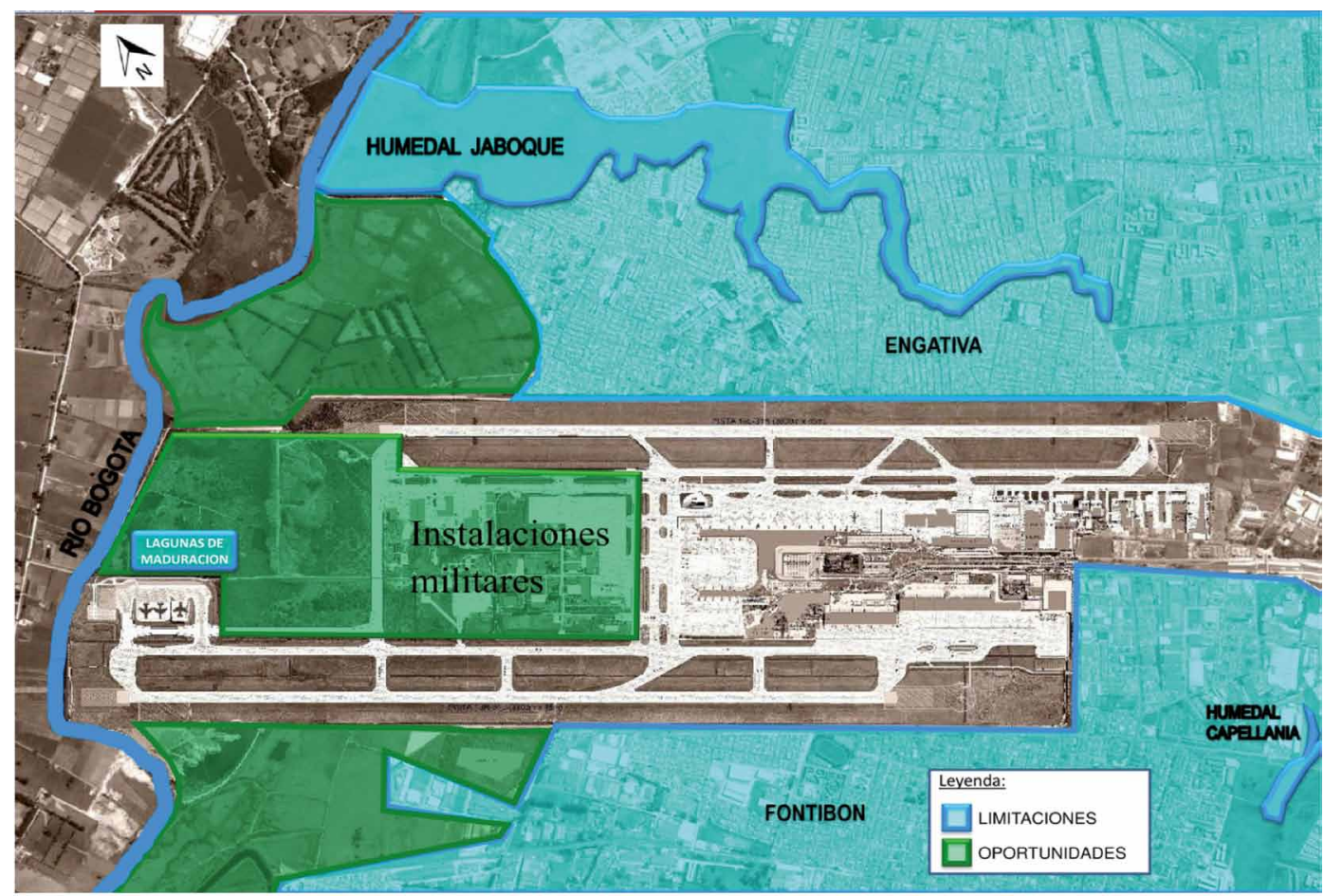

FIG. $7 /$ Disponibilidad de suelo para ampliaciones en el Aeropuerto de Bogotá-El Dorado. 


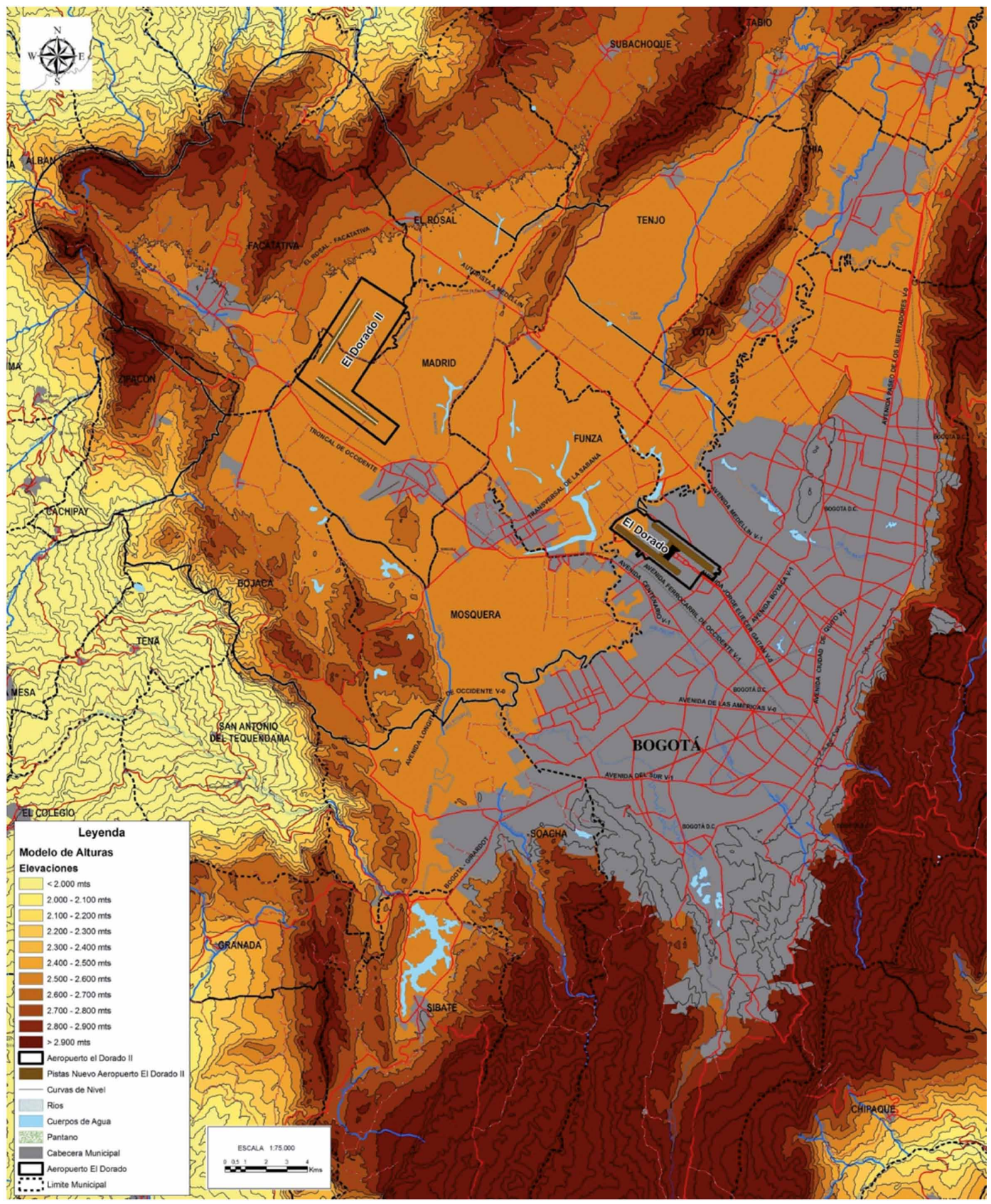

FIG. 8/ Ubicación del próximo (nuevo) Aeropuerto El Dorado II.

Fuente: AEROCIVIL, 2016.

- Como ambos aeropuertos estarán geográficamente muy próximos, es de suponer que las altitudes (con respecto al nivel del mar) de ambos sean muy similares.

- El Dorado II operará en conjunto con El Dorado.
- Las operaciones (despegues / aterrizajes) de El Dorado II deben tener un impacto mínimo en El Dorado.

- Las (dos) pistas paralelas en El Dorado II deben tener una separación de al menos $1.400 \mathrm{~m}$. Se recuerda que a largo plazo el 
sistema de pistas estará conformado por tres pistas, dos de ellas paralelas entre sí, y la tercera perpendicular a los dos anteriores.

- La longitud mínima de pista en El Dorado II deberá ser de $3.800 \mathrm{~m}$. Las longitudes de pista de 4.100 y $4.700 \mathrm{~m}$ pueden satisfacer las cargas de pasajeros típicas de toda la flota (todas las regiones geográficas) al 100\%, mientras que los vuelos a las Américas pueden llevar el $100 \%$ de carga útil típica con cualquiera de las opciones de longitud de pista. Los vuelos transatlánticos plantean los mayores desafíos operacionales con respecto a la longitud de la pista, con solo el $72 \%$ de carga útil típica en una pista de $3.800 \mathrm{~m}$. Sin embargo, la capacidad aumenta hasta el $82 \%$ con una pista de $4.100 \mathrm{~m}$. Una pista de $4.700 \mathrm{~m}$ puede satisfacer todas las cargas útiles típicas al $100 \%$.

Como resultado de los estudios, y considerando las exigencias establecidas, se decidió que la localización óptima del nuevo aeropuerto debería ser al occidente (y a $15 \mathrm{~km}$ ) del actual Aeropuerto El Dorado, en zona rural (mayoritariamente no habitada) de los municipios de Madrid y Facatativá (en la región administrativa de Cundinamarca) y a una altitud de $2.560 \mathrm{~m}$ (ver FIG. 8). El área reservada (actual y futura) para todo el complejo aeroportuario es de 1.980 ha. El desarrollo del futuro aeropuerto contempla la construcción de tres pistas, la primera, con una orientación muy similar o igual a las existentes en el Aeropuerto El Dorado (13/31 o 15/33 o 16/34), para la entrada de operación del aeropuerto (2027/2028), la segunda pista cinco años después y perpendicular (o casi) a la anterior (posiblemente 04/22), y la tercer pista quince años después de la segunda pista (ANI, 2018) y paralela a la segunda pista (es decir de orientación 04/22); todo ello según las proyecciones de la demanda realizadas en 2015 , fechas y tiempos que podrían cambiar si cambian las previsiones de la demanda a realizar una vez el aeropuerto ya esté operando.

\subsection{Escenarios de asignación de la demanda}

Con el fin de establecer el nivel de demanda en el nuevo aeropuerto El Dorado II, Ios planificadores aeroportuarios asignaron pronósticos de pasajeros y operaciones entre El Dorado y El Dorado II. Se consideraron un conjunto de supuestos de reubicación y asignación para cada segmento del tráfico aéreo y se revisaron y analizaron varias opciones de reubicación de aerolíneas comerciales de pasajeros. Las opciones examinadas incluyeron trasladar a todas las aerolíneas que no pertenecen a la alianza Star Alliance, trasladar las aerolíneas nacionales distintas a Avianca (primer operador aerocomercial de Colombia y miembro de Star Alliance), y/o trasladar a los operadores internacionales más pequeños a El Dorado II. En cada escenario, se debían mantener ciertos supuestos preliminares, como eran las prioridades operacionales para El Dorado según lo especificado por la autoridad pública aeronáutica de Colombia. Se examinaron alternativas de reubicación de las aerolíneas, para dar lugar a una demanda de pasajeros a largo plazo en EI Dorado II de entre 4,1 y 14,3 millones de pasajeros en 2041. Los movimientos (despegues/aterrizajes) resultantes en El Dorado II se proyectan de 101.000 a 207.000 operaciones de aeronaves anuales para 2041.

\subsection{Planificación de uso del suelo en el entorno del nuevo aeropuerto}

Para evitar repetir las (malas) experiencias generadas en el uso del suelo y el no-planificado no-ordenado desarrollo urbano en el entorno del actual Aeropuerto El Dorado, tal como se explicó en una sección anterior, ahora, para el desarrollo del nuevo aeropuerto, los planificadores aeroportuarios contemplan en su plan de desarrollo del aeropuerto, a corto, medio, largo y muy largo plazo, lo ya documentado y fijado en los Planes de Ordenamiento Territorial (POTs), en los Planes Básicos de Ordenamiento Territorial (PBOTs) y en los Esquemas de Ordenamiento Territorial (EOTs) de los municipios que conforman el área de influencia del futuro aeropuerto (AEROCIVIL, 2016). Entonces, en la FIG. 9 se muestra la clasificación del suelo, al presente, pero con vigencia futura, en la región donde se situará el nuevo aeropuerto. En dicha figura se pueden apreciar demarcadas tres zonas perimetrales de 3,8 y $13 \mathrm{~km}$ de radio con referencia al centro del polígono de ubicación del nuevo aeropuerto. Estas zonas identificadas como Zona puntual o Zona A (entre los 0 y 3,0 $\mathrm{km}$ del centro del polígono), Zona local o Zona $B$ (entre los 3,1 y $8,0 \mathrm{~km}$ del centro del polígono), y Zona regional o Zona C (entre los 8,1 y 13,0 km del centro del polígono), engloban las principales repercusiones que se pueden dar también en los usos del suelo, incidiendo en la viabilidad urbana del proyecto. Los radios de $3 \mathrm{~km}, 8 \mathrm{~km}$ y $13 \mathrm{~km}$ son estándares internacionales adoptados por la Organización de Aviación Civil Internacional (OACl) para regular los aspectos descritos (OACl, 2002), aspecto también regulado por la autoridad aeronáutica colombiana (AEROCIVIL, 2009). 


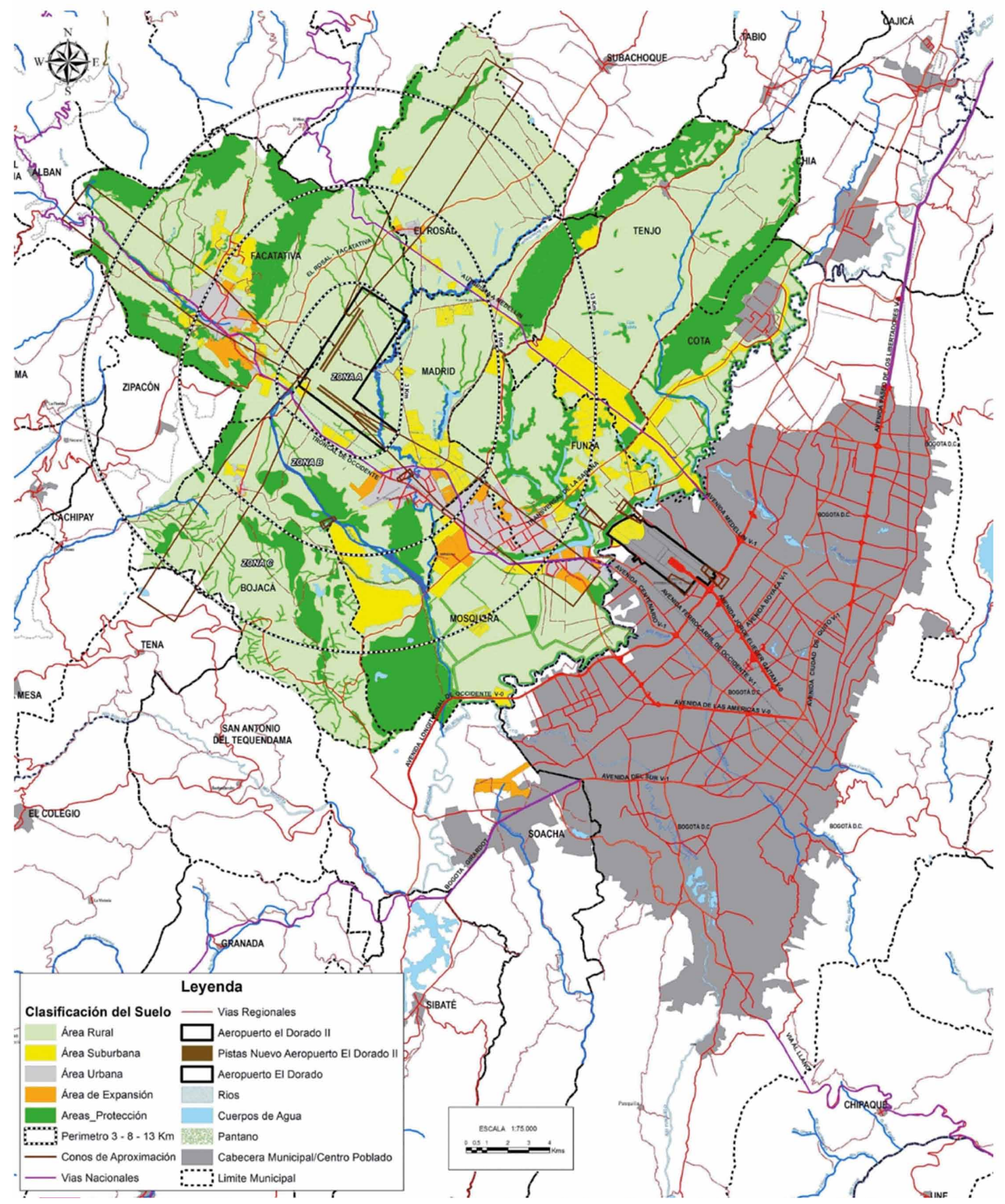

FIG. 9/ Clasificación del suelo en el entorno del próximo (nuevo) Aeropuerto El Dorado II.

Fuente: AerociviL, 2016.

\subsection{Escenarios proyectados de conectividad-movilidad}

Con base en el análisis de viabilidad urbana y en los estudios de conectividad, los planificadores (aeroportuarios y urbanos) desarrollaron algunas alternativas de la conectividad asociada al desarrollo urbano de la región comprendida entre el actual Aeropuerto El Dorado y el futuro Aeropuerto El Dorado II. Estas alternativas hacen parte fundamental de la integración que se requiere entre los dos aeropuertos en términos ambientales, viales, funcionales, espaciales y operacionales para que las dos terminales aéreas operen de tal forma que se consiga optimizar y maximizar la eficiencia del sistema multi-aeropuerto. 


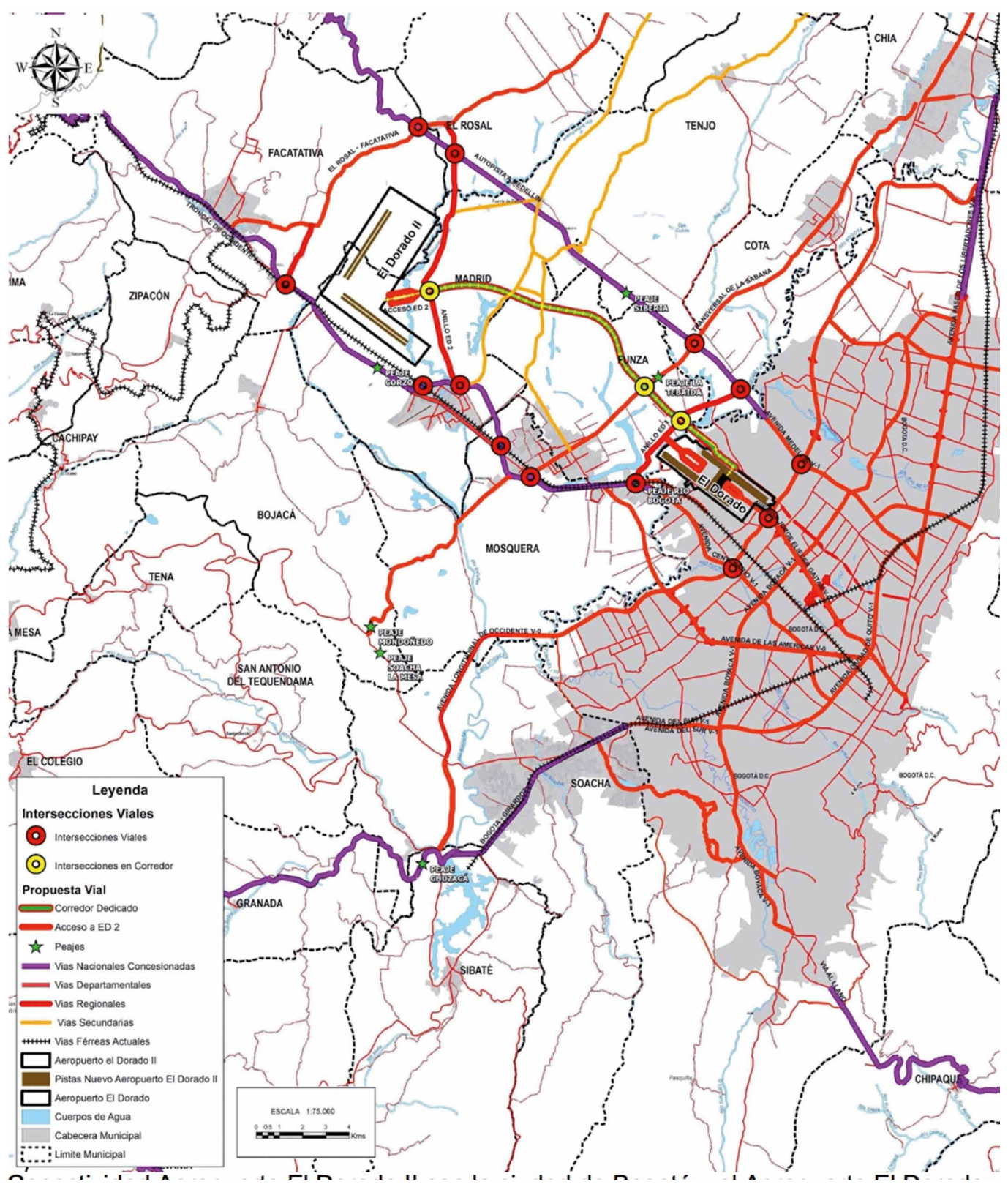

FIG. 10/ Conectividad Aeropuerto El Dorado II con la ciudad de Bogotá y el Aeropuerto El Dorado.

Fuente: AEROCIVIL, 2016b.

El principio fundamental de esta integración es la generación de un sistema de conexión entre los dos aeropuertos mediante un "corredor dedicado", por el cual se movilicen los pasajeros y demás servicios de carga y mantenimiento de manera expresa. La conexión de pasajeros por este corredor se puede generar mediante dos modos de transporte exclusivo (por vías de ser-vicio exclusivas), como son el BRT (Bus Rapid Transit) o un tren de cercanías. Asociado a este corredor dedicado, y para facilitar la apropiada conectividad regional de la ciudad de Bogotá y los municipios aledaños con las dos terminales, se requiere de un sistema de vías y transporte público que han de servir para estructurar el desarrollo urbano asociado y que se generará alrededor 
de las dos terminales que seguramente se convertirán de manera conjunta en un gran polo de desarrollo urbano.

De todas las posibles alternativas analizadas hubo una que cobró más fuerza, por su viabilidad, y la misma está compuesta de dos grandes partes o elementos. Por un lado, dicha alternativa consiste en generar un corredor vial como continuidad de una importante arteria vial de la ciudad de Bogotá (conocida como Calle 63) hacia el occidente de la ciudad, que contenga sobre su eje central el corredor dedicado que sirve para la integración exclusiva de las dos terminales. Este corredor conectaría esta arteria vial (Calle 63) sobre el costado norte del actual aeropuerto El Dorado con el polígono de El Dorado II (ver FIG. 10 , en color amarillo). Este nuevo corredor vial tendría entonces la condición de vía pública de uso mixto, es decir, tanto para transporte público masivo (por ejemplo, BRT), con viales de uso exclusivo, como para transporte privado (todo otro tipo de vehículo, público o privado) sobre viales de uso no exclusivo. El objetivo de esta configuración de corredor dedicado es facilitar las conexiones, cruces e integración, tanto de las asociadas al servicio de las dos terminales aéreas como con las demás vías de la región. El segundo elemento de la alternativa seleccionada es el desarrollo de un tren de cercanías.

Muy recientemente, el 7 de enero de 2020, se firmó el contrato de construcción del (nuevo) tren de cercanías denominado Tren de Cercanías de la Sabana de Bogotá (o RegioTram de Occidente) que conectará la ciudad de Bogotá con los municipios periféricos (pertenecientes a la región administrativa de Cundinamarca), incluidos los municipios donde estará el nuevo aeropuerto (CundinAmARCA, 2020). La red tendrá una estación en el actual Aeropuerto El Dorado y se espera también tenga una estación en el nuevo aeropuerto El Dorado II, o muy próximo al mismo. Mencionar que la infraestructura de red férrea (las vías) ya existe (ver FIG. 10). Se prevé que este tren de cercanías inicie operaciones en el primer trimestre de 2024.

Finalmente, mencionar que ya se está trabajando en la construcción de vías nacionales de alta capacidad (aunque no serán autopistas) con peaje, es decir, son obras viales concesionadas que conectan la ciudad de Bogotá y otras ciudades de la región (y de otras regiones del país). Estas vías nacionales tendrán conectividad con el nuevo aeropuerto. Por otro lado, se prevé mejorar, y construir nuevas vías departamentales principales, la mayoría de las mismas conectarán la ciudad de Bogotá con los municipios periféricos, incluidos los municipios que albergarán el nuevo aeropuerto. También se mejorará la calidad y operatividad de la vías secundarias y municipales que conectan o convergen a los municipios donde se situará el nuevo aeropuerto (ALCALDÍA DE BogotÁ, 2018, ANI, 2018; AerocIVIL, 2016).

\subsection{Planificación ambiental del nuevo aeropuerto y su entorno}

Los estudios de impacto ambiental del proyecto identifican impactos en el medio abiótico y biótico, y para todos ellos se prevé el diseño de "medidas de manejo" con el objetivo de mitigar los impactos previstos (ANI, 2018).

En cuanto a la incidencia del ruido en los usos del suelo, los planificadores del proyecto aeroportuario desarrollaron un "plan de zonificación del ruido" como instrumento que orienta y viabiliza, a largo plazo, una zonificación del uso del suelo adecuado para las áreas afectadas por el ruido aeronáutico. De acuerdo con el manual-guía de protección ambiental para aeropuertos de la $\mathrm{OACl}$ (OACl, 2002), la práctica ha demostrado que tienen que ser consideradas por lo menos dos curvas de ruido, lo que permite delimitar tres áreas de planificación:

- Zona A: es el área más próxima a la pista del aeropuerto y por ello su ambiente es extremadamente ruidoso, pudiendo ocasionar serios problemas de incomodidad en las exposiciones prolongadas y donde la mayoría de las actividades urbanas no son permitidas (uso permitido del suelo: solo industrial).

- Zona B: área donde el ambiente es medianamente ruidoso, registra niveles de incomodidad moderados y gran parte de las actividades urbanas pueden desarrollarse con alguna restricción (uso permitido del suelo: industrial, comercial, de servicios, gubernamental).

- Zona C: comprende el área más distante de la pista donde normalmente el desarrollo de actividades urbanas no sufre restricciones en función del ruido aeronáutico (uso permitido del suelo: todos los tipos, incluido el residencial).

Con base en los resultados de la modelación de un escenario (a largo plazo) con dos pistas, las mismas perpendiculares entre sí, y los valores máximos permisibles de ruido ambiental para los diferentes usos del suelo (según reglamentación local vigente, Resolución 627 del 7 de abril de 2006 (MINAMBIENTE, 2019)) se delimitaron las zonas del plan de zonificación del ruido, las cuales se puede inferir del resultado del modelo de emisión de ruido para el parámetro LAeqD 


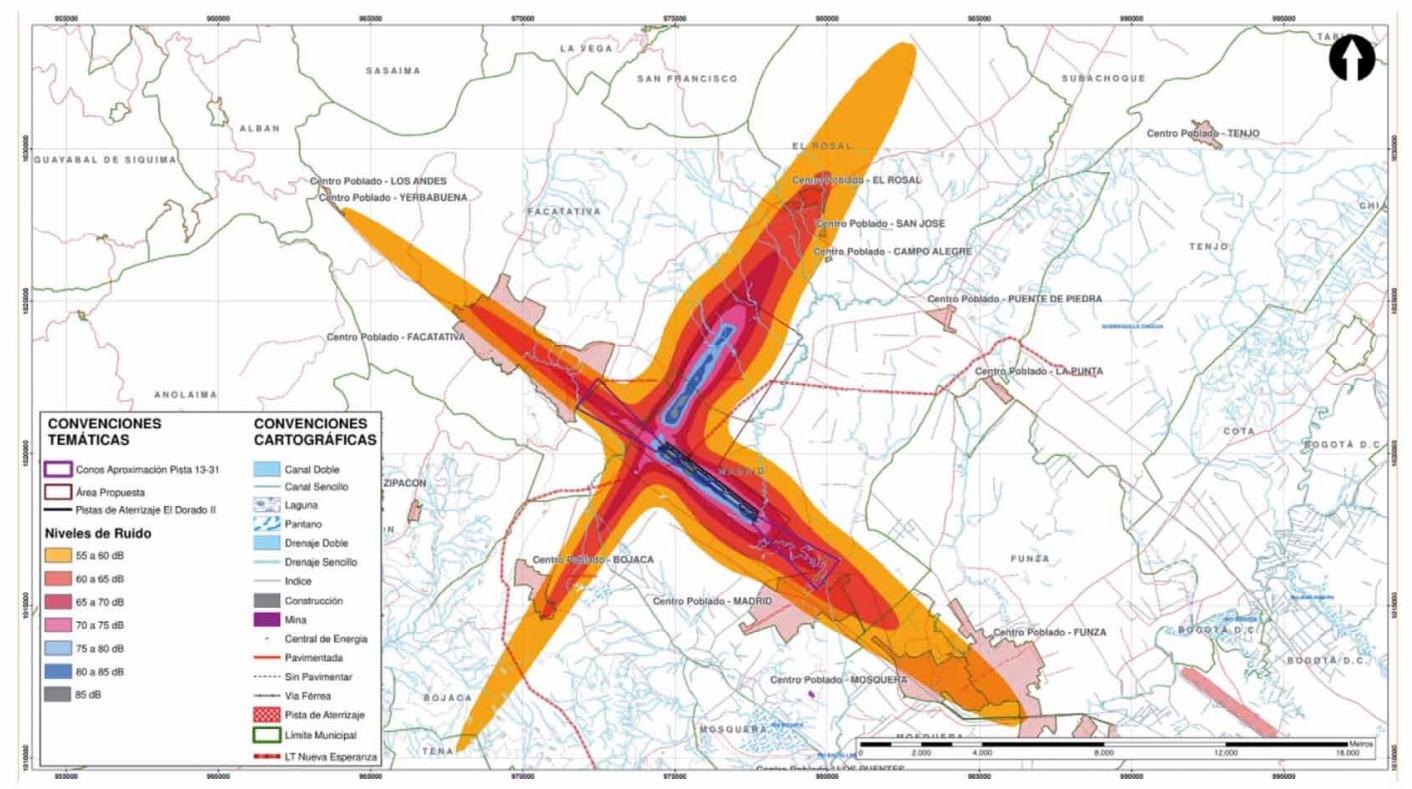

FIG. 11/ Aeropuerto El Dorado II. Modelación de ruido, isófonas LAeqD. Escenario a largo plazo (configuración de dos pistas).

Fuente: AEROCIVIL, 2016.

(nivel sonoro continuo equivalente ponderado A diurno) (ver FIG. 11). La zona A corresponde a la mayor envolvente de las isófonas LAeqD con valores superiores a $70 \mathrm{~dB}$ que se esperan para el año 2041. La zona B está delimitada por la mayor envolvente de las isófonas $L A e q D$ entre 60 y 65 dB que se esperan para el año 2041. La zona $\mathrm{C}$ está delimitada por la mayor envolvente de las isófonas LAeqD entre 55 y $60 \mathrm{~dB}$ que se esperan para el año 2041. Como se aprecia en la FIG. 11, cuatro pequeños municipios se verían parcialmente afectados, pero siempre en las denominadas zonas $\mathrm{C}$ y/o $\mathrm{B}$; ningún municipio se encuentra, en el presente, en la zona A (de mayor presión sonora), y se espera mantener esta situación a largo plazo (2041).

\subsection{Licenciamiento ambiental del proyecto}

A mediados de 2019 la autoridad ambiental colombiana, ANLA (Autoridad Nacional de Licencias Ambientales), suspendió la entrega de la licencia ambiental para el proyecto del nuevo aeropuerto (ANLA, 2019). Dicha decisión fue tomada en vista de que la información suministrada por el gestor público del proyecto, la Agencia Nacional de Infraestructura (ANI) no era suficiente para poder tomar una determinación. La ANLA argumenta que el estudio de impacto ambiental entregada por el gestor público (ANI) para el trámite de solicitud de licencia ambiental para el proyecto Aeropuerto El Dorado II es insuficiente para realizar una evaluación adecuada y generar un pronunciamiento de fondo en relación con la viabilidad o no del referido proyecto. De entre los aspectos más relevantes que fundamentan la no entrega de la licencia ambiental se destacan: a) no presenta la información relacionada a ecosistemas sensibles ni identifica las áreas de afectación directa e indirecta; b) no existencia de información relacionada a aspectos sociales para las áreas de afectación directa e indirecta; y c) deficiencias importantes en la información relacionada con las amenazas naturales que pueden afectar las actividades constructivas y operacionales, con lo cual no es posible tener el conocimiento del riesgo que traería consigo la aprobación de la obra. Esto no significa que se haya negado de manera definitiva la mencionada licencia, eso sí el gestor público del proyecto deberá presentar un nuevo y completo estudio (subsanando todas las deficiencias del primer estudio presentado, identificadas por la ANLA), iniciando así un nuevo proceso de solicitud de la licencia ambiental para el proyecto. Esta circunstancia, sin lugar a dudas, generará un importante retraso en el desarrollo del proyecto del nuevo aeropuerto. 


\section{Conclusiones}

El Aeropuerto de Bogotá-EI Dorado se localizó originalmente en zona prácticamente rural, alejada del centro de la ciudad. Con el tiempo el desarrollo urbano fue creciendo desde el centro de la ciudad hacia al aeropuerto y viceversa, y sobre todo en su inmediato entorno. Ante esta evolución el aeropuerto debería haber servido como articulador de la ciudad (Bogotá) y la región (Departamento de Cundinamarca) pero el análisis aquí presentado acusa que su desarrollo ha estado casi totalmente desligado de su entorno inmediato. Hace aproximadamente cinco décadas, poco tiempo después de la entrada en operación del aeropuerto, en las dos localidades que circundan el aeropuerto, dio inicio una ocupación masiva (mayoritariamente informal y no controlada) del territorio por una población en constante crecimiento y con actividades muy diversas. El área de influencia directa del aeropuerto ha tenido un gran potencial urbano pero que sin la presencia de estrategias y escenarios de planificación regional y de concertación público-privadas no se han logrado resolver problemas para los cuales se necesita una visión holística e integrada del territorio, como el aprovechamiento eficiente del suelo, la disponibilidad y calidad del espacio público y la infraestructura de servicios públicos domiciliarios.

El Aeropuerto de Bogotá-El Dorado ha evolucionado desde una pequeña y modesta infraestructura a una gran instalación que hoy en día conecta la ciudad de Bogotá con el resto del país y el mundo, por lo tanto, con el correr del tiempo ha impactado de manera determinante en el desarrollo económico de la ciudad y su región. Ahora bien, como la proyección de la demanda aquí presentada nos indica, con la capacidad instalada actual es imposible dar respuesta a la demanda proyectada, principalmente a medio, largo y muy largo plazo. Por lo que se torna necesario ampliar la capacidad del aeropuerto, y en el corto plazo. Pero como se ha presentado aquí, razones de tipo ambiental y de no-existencia de suelo disponible, impiden o hacen imposible la ampliación / expansión de las actuales infraestructuras e instalaciones, en especial su sistema de pistas. Esta situación condujo a la autoridad pública aeronáutica de Colombia a poner en marcha el proyecto para un nuevo aeropuerto el cual estará situado en la misma región metropolitana que el actual (con una separación entre ambos de $15 \mathrm{~km}$ ), y que por ahora se conoce como Aeropuerto El Dorado II, constituyendo así un sistema multi-aeropuerto que servirá a la ciudad de Bogotá y zona de influencia.

Las líneas de desarrollo urbano, de conectividadmovilidad y ambiental de la planificación del nuevo aeropuerto aquí presentadas (establecidas para el corto, medio, y largo plazo), invitan a pensar que los planificadores han aprendido de la lecciones y experiencias que dejó el desarrollo histórico del entorno urbano del aeropuerto principal (El Dorado) y motivará a los planificadores, tanto aeroportuarios como los urbanos de las administraciones locales, a la implementación de buenas prácticas para la futura gestión y planificación urbana-territorial del próximo sistema multiaeropuerto El Dorado-El Dorado II.

\section{Bibliografía}

ACl-Airport Council International (2019): Annual World Airport Traffic Report. Montreal: Airport Council International.

Aerocivil (2008): Manual de atenuación de ruido de las aeronaves para el Aeropuerto Internacional El Dorado. Bogotá: Aerocivil.

- (2009): El uso de suelos en áreas aledañas a aeropuertos. Bogotá: Aerocivil.

- (2014a): Actualización del Plan Maestro del Aeropuerto Internacional El Dorado. Bogotá: Aerocivil.

- (2014b): Estudio de impacto ambiental para la modificación de la licencia ambiental el Aeropuerto Internacional El Dorado de la Ciudad de Bogotá. Bogotá: Aerocivil.

- (2016): Análisis técnico de la viabilidad operacional y elaboración del plan maestro aeroportuario para el proyecto de infraestructura El Dorado II. Bogotá: Aeronáutica Civil de Colombia (Aerocivil).

- (2020): Estadísticas de actividades aeronáuticas. http://www.aerocivil.gov.co/atencion/estadisticasde-las-actividades-aeronauticas

AIP (2013): Organización de la afluencia de tránsito aéreo (ENR 1.9). Bogotá: Aerocivil.

- (2019): AIP SKBO-Bogotá EI Dorado. Bogotá: Aerocivil.

Alcaldía de Bogotá (2018): Aeropuerto El Dorado, retos y oportunidades. Perspectiva urbana y regional. Bogotá: Alcaldía de Bogotá.

AlkaAbi, K. \& Debbage, K. (2007): «Air passenger demand and skilled labor markets by US metropolitan area». Journal of Air Transport Management, 13(3), 121-130.

ANI (Agencia Nacional de Infraestructura) (2018): Factibilidad y Estructuración - APP Aeropuerto El Dorado II. Bogotá: Agencia Nacional de Infraestructura.

ANLA (2019): Auto Nº3169, del 17 de mayo de 2019. Bogotá: Autoridad Nacional de Licencias Ambientales de Colombia.

APPoLD, S. \& KASARDA, J. (2013): «The airport city phenomenon: Evidence from large US airports». Urban Studies, 50(6), 1239-1259.

Ashford, N. \& Mumayiz, S. \& WRIGHT, P. (2011): Airport Engineering. New Jersey: John Wiley \& Sons.

BANAI, R. (2017): «The aerotropolis: Urban sustainability perspectives from the regional city». The Journal of Transport and Land Use, 10(1), 357-373.

Bezerra, G. \& Gomes, C. (2019): «Determinants of 
passenger loyalty in multi-airport regions: Implications for tourism destination». Tourism Management Perspectives, 31, 145-158.

Bonnefoy, P. \& Hansman, R. (2007): «Scalability and Evolutionary Dynamics of Air Transportation Networks in the United States». AIAA Air Technology, Integration and Operations Conference, 2007, Belfast (UK).

- P. \& de neufVille, R. \& Hansman, R. (2010): «Evolution and Development of Multi-Airport Systems: A Worldwide Perspective». Journal of Transportation Engineering, 136(11), 1021-1029.

BOQUET, Y. (2018): «From airports to airport territories: expansions, potentials, conflicts». Human Geographies-Journal of Studies and Research in Human Geography, 12(2), 137-156.

BRUECKNER, J. (2002): "Airport Congestion When Carriers Have Market Power». The American Economic Review, 92, 1357-1375.

- (2003): «Air traffic and urban economic development». Urban Studies, 40(8), 1455-1469.

BuDD, L. \& Ison, S. (2017): Air transport management. New York: Routledge.

Burghouwt, G. (2017): «Influencing Air Connectivity Outcomes». Roundtable on Capacity building through efficient use of existing airport infrastructure, 9-10 March 2017, Querétaro.

- G. \& REDONDI, R. (2013): "Connectivity in air transport networks: an assessment of models and applications». Journal of Transport Economics and Policy, 47(1), 35-53.

BUtTON, K. \& TAYLOR, S. (2000): «International air transportation and economic development». Journal of Air Transport Management, 6(4), 209-222.

CCB (2008a): Caracterización urbanística, social y ambiental del entorno del Aeropuerto Internacional El Dorado. Bogotá: Cámara de Comercio de Bogotá.

- (2008b): Aeropuerto El Dorado, una oportunidad para el desarrollo regional. Bogotá: Cámara de Comercio de Bogotá.

CEDINS (2011): Macroproyecto Urbano Regional Aeropuerto EI Dorado - MURA. Componentes, impactos y propuestas sociales. Bogotá: CEDINS.

CEPAL (2019): Airport infrastructure in Latin America and the Caribbean. Bulletin 370.

COOPER, A. \& SMITH, P. (2005): The economic catalytic effects of air transport in Europe. Final report EEC/ SEE/2005/004. Brussel: Eurocontrol.

Cundinamarca (2020): RegioTram. https://efr-cundinamarca.gov.co/es

DANE (2019): Departamento Administrativo Nacional de Estadística. https://www.dane.gov.co/

de NeufVILle, R. (1984). «Planning for Multiple Airports in a Metropolitan Region». Built Environment, 10(3), 159-167.

- (1995): «Management of multi-airport systems. A development strategy». Journal of Air Transport Management, 2(2), 99-110.

- \& Odoni, A. (2013) : Airport Systems, Planning, Design, and Management. New York: McGrawHill.

Díaz Olariaga, O. (2016): «Análisis del desarrollo reciente del transporte aéreo en Colombia». Revista Transporte y Territorio, 14, 122-143.

- (2017): «Políticas de privatización de aeropuertos.
El caso de Colombia». Documentos y Aportes en Administración Pública y Gestión Estatal, 29, 7-35. - (2018): «Análisis de mitigación de ruido aeroportuario. El caso del Aeropuerto Internacional de Bogotá-El Dorado (Colombia)». Ciudad y Territorio, 197, 557-576.

— \& ÁvILA J. (2015): «Evolution of the airport and air transport industry in Colombia and its impact on the economy». Journal of Airline and Airport Management, 5(1), 39-66.

— \& Rodriguez, Y. \& Muñoz, C. (2018a): «Análisis de perfil de usuario y de accesibilidad al aeropuerto». $X X$ Congreso Panamericano de Ingeniería de Tránsito, Transporte y Logística, 26-28 Septiembre 2018, Medellín (Colombia).

— \& GaVILÁn, A., \& Ortiz, C. (2018b): «Accesibilidad vial al aeropuerto. El caso del Aeropuerto de Bogotá-EI Dorado». XX Congreso Panamericano de Ingeniería de Tránsito, Transporte y Logística, 26-28 septiembre 2018, Medellín (Colombia).

DNP (2007): CONPES 3490-Estrategia institucional para el desarrollo del macroproyecto urbano regional del Aeropuerto El Dorado de Bogotá. Bogotá: Dirección Nacional de Planeación.

Fasone, V. \& Giuffre, T. \& Maggiore, P. (2012): «Multi-Airport System as a Way of Sustainability for Airport Development: Evidence from an Italian Case Study». Procedia-Social and Behavioral Sciences, 53, 96-105.

Figueroa, O. \& RozAs, P. (2005): Conectividad, ámbitos de impacto y desarrollo territorial: el caso de Chile. Santiago de Chile: CEPAL.

Fuellhart, K. \& O'connor, K. \& Woltemade, C. (2013): «Route-level passenger variation within three multi-airport regions in the USA». Journal of Transport Geography, 31, 171-180.

GaWC (2019a): Globalization and World Cities Research Network. https://www.lboro.ac.uk/gawc/

- (2019b): The World According to GaWC 2018. https://www.lboro.ac.uk/gawc/world2018t.html

GoETZ, A. (1992): «Air passenger transportation and growth in the U.S. urban system, 1950-1987». Growth and Change, 23, 218-242.

Green, R. (2007): "Airports and economic development». Real Estate Economics, 35(1), 91-112.

Horonjeff, R. \& al. (2010): Planning and Design of Airports. New York: McGrawHill.

IATA (2019): World Air Transport Statistics. Geneva: IATA.

ICAO (2008): Guidance on the Balanced Approach to Aircraft Noise Management. Montreal: ICAO

JANIC, M. (2009): Airport analysis, planning and design: demand, capacity and congestion. New York: Nova Science Publishers.

Kazda, A. \& Caves, R. (2015): Airport design and operations. Bingley: Emerald.

KnipPenberger, U. \& Wall, A. (2010): Airports in Cities and Regions. Karlsruhe: KIT Scientific Publishing.

Liv, Z. \& Debbage, K. \& Blackburn, B. (2006): «Locational determinants of major US air passenger markets by metropolitan area». Journal of Air Transport Management, 12, 331-341.

MARTIN, J.C. \& Voltes-DorTA, A. (2011). «The dilemma between capacity expansions and multi-airport systems: empirical evidence from the industry's 
cost function». Transportation Research, 47(3), 382-389.

Minambiente (2019): Resolución 627 del 7 de Abril de 2006. Bogotá: Ministerio de Ambiente y Desarrollo Sostenible (Colombia).

MUN, S. \& TERAJI, Y. (2012): «The organisation of multiple airports in a metropolitan area». Journal of Transport Economics and Policy, 46(2), 221-237.

NASA (2011): Investigating the nature of and methods for managing metroplex operations. Hanover (MD): NASA Center for AeroSpace Information.

NEAL, Z. (2012): «Creative employment and jet set cities: disentangling causal effects». Urban Studies, 49, 2693-2709.

OACI (2002): Manual de Planificación de Aeropuertos. Parte 2: Utilización del terreno y control del medio ambiente. Montreal: OACI.

Pels, E. \& Verhoef, E. (2004): «The Economics of Airport Congestion Pricing». Journal of Urban Economics, 55, 257-277.

Postorino, M. (2010). Development of Regional Airports. Southampton: WIT Press.

- \& Pratico, F. (2012): «An application of the MultiCriteria Decision-Making analysis to a regional multi-airport system». Research in Transportation Business \& Management, 4, 44-52.

RozAs, P. \& FIGUEROA, O. (2006): Conectividad, ámbitos de impacto y desarrollo territorial: análisis de ex- periencias internacionales. Santiago de Chile: CEPAL.

SÁNCHEZ, G. A. (2011): «Incidencia del aeropuerto Eldorado en la estructura espacial de la ciudad de Bogotá, periodo 1990-2010». Perspectiva Geográfica, 16, 173-196.

SDM (2015): Informe de Indicadores Encuesta de Movilidad de Bogotá. Bogotá: Secretaria Distrital de Movilidad, Alcaldía de Bogotá.

Van De Vijver, E. \& Derudder, B. \& Witlox, F. (2016): «Air passenger transport and regional development: cause and effect in Europe». Promet - Traffic \& Transportation, 28(2), 143-154.

VASIGH, B. \& Fleming, K. \& TACKER, T. (2013): Introduction to Air Transport Economics. Burlington: Ashgate Publishing.

ZAK, D. \& GetZneR, M. (2014): «Economic effects of airports in Central Europe: A critical review of empirical studies and their methodological assumptions». Advances in Economics and Business, 2(2), 100-111.

ZhANG, A. \& ZhANG, Y. (2006): «Airport Capacity and Congestion When Carriers Have Market Power». Journal of Urban Economics, 60, 229-247.

Zietsman, D. \& VANDERSChUREN, M. (2014): «Analytic Hierarchy Process assessment for potential multiairport systems - The case of Cape Town». Journal of Air Transport Management, 36, 41-49. 